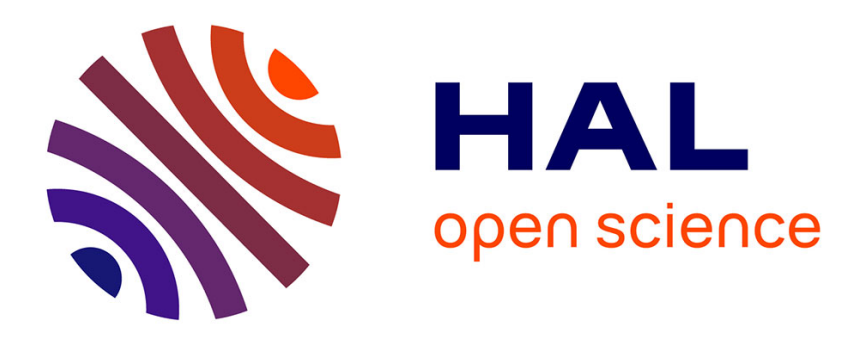

\title{
AL-MAQĀMA AL-BIŠRIYYA UNE ÉPOPÉE MYSTIQUE
}

\author{
Katia Zakharia
}

\section{To cite this version:}

Katia Zakharia. AL-MAQĀMA AL-BIŠRIYYA UNE ÉPOPÉE MYSTIQUE. Arabica, 1990. halshs01270252

\section{HAL Id: halshs-01270252 \\ https://shs.hal.science/halshs-01270252}

Submitted on 14 Feb 2016

HAL is a multi-disciplinary open access archive for the deposit and dissemination of scientific research documents, whether they are published or not. The documents may come from teaching and research institutions in France or abroad, or from public or private research centers.
L'archive ouverte pluridisciplinaire HAL, est destinée au dépôt et à la diffusion de documents scientifiques de niveau recherche, publiés ou non, émanant des établissements d'enseignement et de recherche français ou étrangers, des laboratoires publics ou privés. 


\title{
AL-MAQĀMA AL-BIŠRIYYA UNE ÉPOPÉE MYSTIQUE
}

\author{
PAR KATIA ZAKHARIA
}

\begin{abstract}
Celui qui en reste à l'histoire pénètre dans quelque chose d'opaque dont il ne se rend pas compte, et celui qui s'en tient à la signification ne peut rejoindre l'obscurité dont elle est la lumière dénonciatrice. Les deux lecteurs ne peuvent jamais se rattraper, on est l'un, puis l'autre, on comprend toujours plus ou toujours moins qu'il ne faut. La vraie lecture reste impossible.

Maurice Blanchot, La Part du Feu.
\end{abstract}

Bišr Ibn 'Awāna al-'Abdiyy était un gueux, il attaqua un « convoi de voyageurs parmi lesquels se trouvait une belle femme qu'il épousa $»^{1}$. Ainsi commence, de façon somme toute banale, al-maqāma al-bišriyya de Badī‘ al-Zamān al-Hamad̄ānī (968-loog).

Cet article se propose de réhabiliter, par-delà le caractère héroïque et amoureux de cette fiction d'allure mythique, l'hypothèse à ce jour prudemment écartée sans avoir été sérieusement examinée, selon laquelle il serait hautement plausible que les maqāma-s, certaines d'entre elles du moins, fussent l'expression ésotérique de courants religieux ou politico-religieux ${ }^{2}$.

Il ne s'agit pas ici de céder aux charmes surannés d'une vision syncrétiste, par définition réductrice, mais de mettre à l'épreuve une hypothèse que l'ancrage culturel de ce texte et la biographie de son auteur, connu pour ses accointances avec le milieu ismaïlien³, engagent à explorer attentivement.

Il est certain que les questions suscitées à ce propos par le genre maqāma, sont loin d'être élucidées. La coexistence, un siècle encore après la mort de Hamadānī, sous le même titre : Maqāmāt de deux œuvres aussi différentes en apparence que celle de Ḥarīrī (habituellement

\footnotetext{
${ }^{1}$ Pour la commodité de la lecture, le texte arabe des citations n'apparaîtra dans le corps de l'article ou en note que lorsqu'il sera particulièrement bref ou immédiatement nécessaire à notre propos. Pour le reste, l'ouvrage de référence est: al-Hamadānī, Maqāmāt, qaddama lahā wa-šaraha ġawāmị̣ahā al-'allāmatu aš-šayhu Muḥammad 'Abduh, 7e édition (1ère édition 1889), Dār al-Mašriq, Beyrouth, 1973, 285 p. Al-maqāma al-bišriyya, pp. 250-258. L'ouvrage sera désigné par l'abréviation $m q$., suivie de la page. Ici : $m q$., p. 25 o.

${ }^{2}$ Toute ma reconnaissance va à Monsieur André Roman dont l'intérêt soutenu pour cette hypothèse m'a permis d'en mener à bien l'examen. Je remercie également Monsieur Abdallah Cheikh-Moussa pour l'attention qu'il a bien voulu porter à ce texte.

${ }^{3}$ Margoliouth D. S., « Al-Hamadhānī », EI, I, pp. 257-258. Blachère R., « Al- Hamadhānī, EF' I, pp. 108-109.
} 
considérée comme un recueil d'historiettes plagiant Hamad̄ān̄̄̄ ${ }^{4}$ et celle de Zamahšarā (monologues religieux $)^{5}$ est l'un des arguments qui plaident favorablement pour une relecture de ces documents a la lumière de notre hypothèse.

C'est dans cette perspective que nous examinerons al-maqāma al- bišriyya, écartant de notre propos, malgré son intérêt, l'analyse des caractéristiques stylistiques et rhétoriques qui pourrait, à elle seule, faire l'objet d'un article. À partir de cette maqāma, pièce d'anthologie renommée surtout pour les vingt-quatre vers dans lesquels le héros décrit son combat avec le lion Don ${ }^{6}$, texte qui ne présente qu'un seul des traits formels qui devaient ultérieurement définir le genre? ${ }^{7}$ il s'agira d'établir comment le faisceau convergent des symboles, la cohérence structurelle et les jeux polysémiques orientes présentent notamment des analogies récurrentes avec Le Livre du Sage et du Disciple. Ce roman initiatique ismaïlien écrit entre le IXe et le Xe siècles est une épopée à caractère mystique remarquablement analysée par Henry Corbin, et dont l'auteur est incertains.

Nous nous devons de préciser dès à présent que ce sont ces analogies qui, une fois identifiées, nous ont permis de percevoir dans la maqāma une cohérence qui, a plus d'un endroit, paraissait lui faire défaut. Elles nous ont permis d'organiser de façon satisfaisante les indices que livrait le texte de Hamaḍānī et de choisir, parmi plusieurs lectures potentielles - allant du mythe au rêve en passant par l'épopée héroïque - celle que nous proposons ici. Il convient que nous précisions également que le choix du Livre du Sage et du Disciple tient, d'une part, à son caractère indubitable de roman initiatique ismaïlien et, d'autre part, aux analogies explicites qu'il établit entre la geste héroïque (qui correspond, dans al-maqāma al-bišriyya, à la réhabilitation sociale d'un « gueux » et l'épopée mystique.

Nous n'ignorons pas que cette lecture d'al-maqāma al-bišriyya ne manquera pas de susciter

\footnotetext{
${ }^{4}$ Al-Harīīi, Les Séances de Hariri publiées en arabe (...) par S. de Sacy, éd. Silvestre de Sacy Antoine Isaac, Paris, Imp. Royale, 1843-53 ; rep. Oriental Press, Amsterdam, l968, 2 vol.

${ }^{5}$ Al-Zamahšarī, Al-Maqāmāt, šarḥu maqāmāti z-Zamahšarāi, éd. Yūsuf Biqāìi, Dār al-Kitāb al-Lubnānī, Beyrouth, 1981, $329 \mathrm{p}$.

${ }^{6}$ Pour la traduction de $d \bar{a} \underline{d}$ par Don, voir $§$ l-5-3 ci-dessous.

${ }^{7}$ La phrase inaugurale commune à la plupart des maqāma-s : « haddațanā 'T́sa bnu Hišām qāla», mq., p. 25o. Signalons qu'une édition au moins de l'ouvrage d'al- Hamadānī, Maqāmāt, éd. Matba at al-Ğawa'ib, Constantinople, 1298 H, propose : « ḩaddațanā al-Hasan Ibn Muhammad al-Fārsīn̄̄». Cette édition classe d'ailleurs Al-maqāma al-bišriyya avec d'autres « histoires plaisantes rapportées par Hamadānī » dans un chapitre faisant suite au corpus des maqāma-s.

${ }^{8}$ Corbin Henry, «Un roman initiatique ismaélien du X ${ }^{\circ}$ siècle » in Cahiers de civilisation médiévale XV/57, pp. 1-25; 58, pp. 121-142, 1972.
} 
chez certains un scepticisme indulgent, réserve ou polémique. Il convient donc que nous commencions par évoquer les différents arguments qui, à notre sens, la légitiment. Comme nous l'avons indiqué, l'auteur a été, selon ses biographes, en contact prolonge avec les Ismaïliens ; certains affirment même que c'est durant cette période de sa vie qu'il entreprit de composer ses maqāma-s.

D'autre part, les principaux indices que nous entreprendrons d'exposer montrent qu'une lecture limitée au texte manifeste d'al-maqāma al-bišriyya, constituerait une approche réductrice, comme le serait d'ailleurs celle qui prétendrait estomper le sens obvie.

À l'évidence nous n'avons pas sélectionné ces indices en vue de les conformer à notre hypothèse. Bien au contraire, nous avons examiné avec minutie l'ensemble des différentes significations de chacun des termes retenus, voire d'autres termes, en raison de leur récurrence ou de leur position. Nous avons constaté alors que si certains vocables présentaient isolement une intéressante polysémie ou quelques liens inattendus avec d'autres éléments du texte, nous en restions chaque fois, exception faite du champ sémantique de l'ontologie mystique ou des symboles ismaïliens, à des points épars, atomises, ne présentant ni organisation ni continuité. Nous en avons déduit que seuls les éléments persistants et structures de notre corpus constituaient des indices fiables.

Ces deux arguments prennent toute leur mesure inscrits dans leur contexte culturel, contexte dans lequel Le Livre du Sage et du Disciple a particulièrement retenu notre attention, en raison de son caractère didactique. En effet, les éléments qui constituent le code ésotérique évoqué dans cet ouvrage sont le plus souvent présentes conjointement avec leur interprétation. Comme le souligne Henry Corbin, ce type de texte «constitue quelque chose d'unique $»^{9}$ et « nous ne connaissons jusqu'ici aucun texte de la littérature ismaélienne ou shi 'ite qui lui soit vraiment comparable $»^{10}$, dans le sens où «ces récits d'initiation personnelle en prose représentent un genre littéraire qui ne s'est développe ni en arabe ni en persan ${{ }^{\mathrm{n}}}$. Peu importent ici les raisons (sociales selon certains, métaphysiques pour H. Corbin) qui expliquent ce phénomène culturel, « ce qui intéresse avant tout, c'est la geste des héros (...),

${ }^{9}$ Corbin H., op. cit., p. 140

${ }^{10}$ Ibid

${ }^{11}$ Ibid. 
le passage de l'épopée héroïque à l'épopée mystique. Le lieu de cette épopée est le mundus imaginalis; elle n'est point constituée de «fiction » mais de faits et d'évènements ayant leur réalité propre, de plein droit, dans le monde imaginal $\gg^{12}$.

Pour H. Corbin, cela implique qu'il convient de ne pas aborder ces textes et les éléments qui les constituent en tant qu'allégories. Bien que nous souscrivions à la fine description qu'il propose lorsqu'il s'agit de définir ce que ces textes sont pour ceux dont ils expriment l'expérience, il nous parait néanmoins nécessaire de dissocier l'examen d'un texte et les « faits (...) ayant leur réalité propre (...) dans le monde imaginal », c'est-à-dire les données appartenant à l'étude ontologique de la foi ou de la vérité.

L'existence d'un document, fut-il aussi rare que Le Livre du Sage et du Disciple, prouve d'ailleurs par elle-même, qu'avant de devenir des faits pour ceux qui y adhèrent, ces éléments présupposent un accord minimal des adeptes en ce qui concerne le codage des signifiants utilisés, en vue de les associer à d'autres signifiés que ceux reconnus par l'usage ; codage sans lequel il n’y aurait ni ne pourrait y avoir discours ésotérique mais simplement délires individuels.

Tout cela n'est pas pour simplifier la tâche des lecteurs. Une fois le code établi et reconnu par les initiés, il devient effectivement possible aux auteurs de passer de l'ordre de l'allégorie à celui du fait. Ayant par conséquent été diffusés codés, sans que le code permettant de les déchiffrer ait été offert simultanément au lecteur, bon nombre de textes ont pu glisser dans le champ de la littérature, se trouver définis rétrospectivement comme des documents littéraires et, pour certains d'entre eux, connaître à ce titre la postérité.

Enfin, il ne faut pas omettre de situer le problème que soulève le codage des textes ésotériques arabes dans un cadre plus vaste. Comment ne pas évoquer le cycle épique de Parsifal, la quête du Graal ou les voyages de Saint Brandan, sans oublier, bien sûr, les aventures de Sindbad ? La question n'est pas d'imaginer quelque corrélation historique car « autant que nous sachions, il n'y en a pas eu ${»^{13}}^{3}$ mais d'insister sur le fait qu'il y a là une démarche fréquente des gnostiques à la recherche de la gnose ; démarche à la suite de laquelle « il arrive que le sabot tombe dans l'empreinte du sabot $»^{14}$.

\footnotetext{
${ }^{12}$ Corbin H., op. cit., p. 141.

${ }^{13}$ Corbin H., op. cit., p. 142.

${ }^{14}$ Proverbe arabe cité notamment par al-Ghazālī et repris par Louis Massignon, à propos de ces hasards bien particuliers.
} 
Après avoir défini les perspectives dans lesquelles nous avons abordé cette lecture, nous allons maintenant examiner les indices textuels sur lesquels elle se fonde.

1. Les indices

1.1. Le récit manifeste

Le texte de Hamad̄ānī se compose de quarante vers et, dans les éditions arabes contemporaines courantes ${ }^{15}$, d'une quarantaine de lignes en prose. Concis, rapide, il est si dense qu'aucun résumé ne saurait en rendre valablement compte.

Rappelons toutefois qu'à peine marié, Bišr apprend que sa cousine germaine Fāțima est, aux dires mêmes de son épouse, le parangon de l'humaine beauté. Bouleversé, il abandonne aussitôt sa femme et fait demander à son oncle la main de sa fille. Éconduit, il se venge en harcelant sans relâche le clan de celle qu'il convoite. L'oncle, résolu à se débarrasser de ce neveu importun, l'envoie quérir une dot de mille chamelles auprès de la tribu de Huzā'a. Pour y parvenir, Bišr doit emprunter un chemin périlleux gardé par le lion Don et le serpent Courageux. Le héros s'y engage, rencontre le lion, le tue et décrit son combat dans un poème qui parviendra à son oncle. L'oncle saisi de remords quand il reçoit ces vers, rejoint son neveu qui, en sa présence, affronte le serpent et le tue ; il accorde alors à Bišr la main de Fāṭima sans qu'il soit plus question de dot. Sur le chemin du retour, Bišr triomphant rencontre un adolescent inconnu et se bat avec lui. Vaincu, mais épargné à deux reprises par le jeune homme qui lui apprend qu'il n'est autre que son fils, il renonce à épouser sa cousine pour la donner en mariage à cet héritier inattendu.

1.2. L'objet de la quête de Bišr

Prise au sens propre, l'intrigue apparente, qui fait du mariage de Bišr avec sa cousine germaine Fāṭima l'objet de la quête du héros, contredit le dénouement du récit dans lequel Bišr renonce à cette belle femme, conquise par lui au péril de sa vie. Il convient donc de chercher à préciser quel enchaînement textuel justifie pleinement ce renoncement en apparence incompréhensible.

La démarche qu'entreprend Bišr a pour origine sa découverte de la beauté de Fāțima. Selon

\footnotetext{
${ }^{15}$ mq. : 170 x 245 ; même format pour l'édition commentée par 'Abd al-Ḥamīd Muhammad Muhyi al-Dīn, Dār al-Kutub al-'ilmiyya, Beyrouth, s.d. (1re édition 1923).
} 
certains manuscrits, cette découverte tiendrait à la curiosité du héros. À peine marié, il aurait en effet demandé à son épouse : «As-tu vu plus belle que toi ?» ('a-ra'ayti aḥsana minki). Celle-ci lui aurait, à cette occasion, appris l'existence de Fāțima. Selon d'autres manuscrits, Bišr se serait contenté de dire à son épouse : «Je n'ai jamais vu de jour semblable à celui-ci » (mā ra'aytu ka-l-yawmi). Ironisant sur l'enthousiasme qu'il manifestait, elle lui aurait révélé l'existence de sa belle cousine. Les deux versions s'accordent cependant à faire passer très rapidement la beauté de Fāțima au second plan : il n'en sera plus question dans la suite du texte.

L'épouse de Bišr lui apprend qu'il est le cousin germain de cette femme exceptionnelle et lui, affirme qu'il consacrera désormais son existence à réhabiliter son honneur :

«Bišr dit: "Malheureuse ! De qui parles-tu là ?"-, Elle répondit: "De ta cousine germaine, la fille du frère de ton père, Fāțima". Puis il dit : "Est-elle aussi belle que tu le dis ?". Elle répliqua : "Plus encore ; bien, bien davantage !". Alors il se mit à déclamer : "Malheur à toi, belle aux blanches dents !/Je ne me figurais pas te

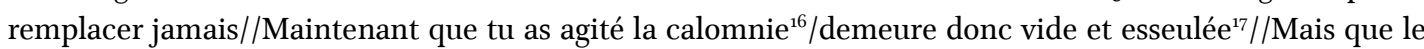
sommeil ne ferme plus mes paupières/avant que je n'aie relevé mon honneur tombé aussi bas que le sol". $»^{18}$

Si l'on cherche à déterminer ce qui, de l'éclat de Fāțima, de l'honneur de Bišr ou du croisement des deux, va dès lors mouvoir le héros, on se trouve rapidement devant une impasse. En effet, si l'on admet que ce mariage, idéalement conforme au schéma social conventionnel, est vraiment l'objectif visé, on ne peut qu'être surpris par le silence que Bišr oppose à son oncle lorsqu'il lui dit : «J'ai fait le serment de ne donner en mariage ma fille, celle-cíi, qu'à celui qui lui ramènera mille chamelles en dot $»^{20}$. L'oncle aurait-il d'autres filles à marier ? Peut-être

\footnotetext{
${ }^{16} \mathrm{M}$. 'Adbuh et 'Abd al-Hamīd prennent le terme ta 'rị̣̂d dans le sens de « calomnie » et comprennent la phrase lawwaḥti bi-t-ta 'rị̣ comme « tu as agité la calomnie ». Ils considèrent l'un et l'autre qu'en parlant à Bišr de sa cousine, l'épouse sous- entend qu'il a failli à son devoir social en ne recherchant pas un mariage consanguin ; que, de ce fait, elle l'insulte. Ta 'rị̣ signifie aussi « allusion » (LA, II, 'araḍ, p. 744; wa-t-ta 'rịdu hilāfu t-taṣrīh) ; singulièrement, « se déclarer à une femme par allusion » (LA, II, p. 744 : at-ta'rị̄̂ (...) 'an yatakallama bi-kalāmin šibhi huitbațihā wa-lā yuṣarriḥa bihi wahuwa-'an yaqūla lahā 'innaki ğamīla (...)). Il nous semble intéressant de signaler que, de ce fait, lawwahti bi-t-ta'rị̄̂peut également être traduit par « tu as [par tes propos] donné tant d'éclat à l'allusion ».

${ }^{17}$ Derrière une simplicité apparente, la traduction de ḩalawti ğawwan fa-ṣfirī wa-bị̣̂i n'est pas sans susciter quelques difficultés. Cet énoncé a été interprété comme le doublet de l'expression lexicalisée halā laki l-ğawwu fa-bị̣̂i wa-ṣfirī, expression quasi-proverbiale attribuée au poète Țarafa par Ibn Manzūr ( $L A$, I, halawa, p. $539 \mathrm{C}_{7}$ et à Kulayb Ibn Rabī’a (Kulayb Wā’il) par 'Abd al-Ḥamīd (op. cit., p. 475, note l). La différence syntaxique entre halā laki l-ğawwu et hualawti ğawwan ne saurait manquer d'induire une différence sémantique. «Demeure vide et esseulée » est une lecture provisoire, en attendant une traduction qui rende mieux compte des particularités syntaxiques de l'énoncé et des résonances dans le contexte de la polysémie des verbes șafara et bāḍa

${ }^{18} \mathrm{mq}$. p. 251.

${ }^{19}$ Souligné par nous. Si la construction arabe 'ibnati hādihi n'implique pas nécessairement une volonté de mise en relief du démonstratif hādihi(celle-ci), il n'en demeure pas moins que la présence de ce démonstratif suscite une interrogation quant à l'éventuelle existence des sœurs de Fāțima.
}

${ }^{20} m q .$, p. $25^{2 .}$ 
dans d'autres conditions? Si Bišr poursuivait seulement le projet de restaurer son honneur en se soumettant à un usage (épouser une cousine germaine), on s'attendrait à ce qu'il s'enquière du sens de cette remarque. Dès lors qu'il n'en fait rien, le propos de ce gueux ne paraît pas être l'obtention d'une réhabilitation sociale par un mariage conforme aux normes.

Si l'on suppose que l'objectif de Bišr est la conquête de Fāțima, cette femme et nulle autre, en raison de sa beauté ou, tout à la fois, de sa beauté et de sa qualité de cousine germaine, on comprend difficilement qu'au moment ou plus aucun obstacle ne l'en sépare, Bišr renonce à ce mariage en faveur de son fils. L'étrangeté de ce renoncement s'accroît d'ailleurs du fait que c'est le héros lui-même, et non son oncle, qui selon le texte, « donna en mariage sa cousine à son fils », le rôle de l'oncle en ce qui concerne le mariage de sa propre fille, indispensable selon le code social, s'étant ainsi « inexplicablement » estompé.

L'absence dans la structure manifeste du texte d'un fil conducteur qui donnerait sens au fait que Bišr, tout à la fois, engage sa vie pour la conquête de Fāțima et renonce à elle de son plein gré, conduit à examiner cet apparent paradoxe : la démarche de Bišr a précisément pour objectif le renoncement à Fāțima en faveur de son fils. Ce paradoxe prend sens dès lors que l'on admet que ce synopsis tire sa cohérence d'une dimension allégorique dans laquelle le mariage avec Fāțima désigne l'accès au savoir de la gnose. Comme l'explique le Sage à son disciple : «En vérité, la Religion (Dīn au sens ismaélien du mot) comporte une clef qui la rend licite ou illicite, pareillement à la différence qu'il y a entre la débauche et le mariage ${ }^{21}$ et « cette clef, c'est l'entrée dans la confrérie ismaélienne ${ }^{22}$.

Répétons-le, il va de soi que notre hypothèse ne tient que parce que le document est jalonné de nombreux indices venant la corroborer. Il ne sera pas possible, en raison de la densité de cette maqāma d'examiner dans le détail chacun de ces indices. C'est pourquoi seuls le seront ceux qui confirment la dimension allégorique ; ceux qui, de façon patente, apparentent cette allégorie à l'ismaïlisme. Nous établirons pour commencer comment dans ce texte le temps est une donnée «existentielle» et non «chronologique ${ }^{23}$; comment l'espace n'y est pas géographique mais symbolique ; comment cette symbolique s'étend à la monture du héros et aux noms propres des personnages entre lesquels divers réseaux d'analogies tissent des liens

\footnotetext{
${ }^{21}$ Corbin H., op. cit., p. 7 .

${ }^{22}$ Ibid.

${ }^{23}$ Corbin H., op. cit., p. 141.
} 
par le truchement d'éléments sémantiques ou syntaxiques. Nous examinerons ensuite les relations qu'entretient le texte dans sa totalité avec les grands symboles du ši ísme ismaïlien ; ce qui permettra, pour finir, d'esquisser une lecture d'al-maqāma al-bišriyya grâce notamment aux éléments d'explication que Le Livre du Sage et du Disciple révèle en ce qui concerne le cheminement initiatique et les codes des ésotéristes. Il nous faut signaler que le caractère particulier de l'hypothèse examinée ici nous engage à n'aborder vraiment la présente lecture qu'après avoir proposé au lecteur l'ensemble des indices qui l'autorisent.

1.3. Le temps de la Quête

À l'heure actuelle, il est d'usage lorsque l'on s'interroge sur la temporalité dans les textes arabes anciens, d'en justifier les spécificités en référence à des paramètres culturels différentiels; il convient donc que nous précisions, pour éviter toute ambiguïté, que les questions qui se posent ici sont d'un autre ordre. En effet, c'est à l'intérieur de la maqāma telle qu'elle s'inscrit dans la culture qui la sous-tend et qu'elle exprime, que l'a-temporalité qui caractérise tous les événements du récit s'oppose à la durée cristallisée en une seule phrase, celle de l'oncle disant aux hommes du clan : «Accordez-moi un délai ${ }^{24}$ afin que je le fasse périr par quelque moyen $»^{25}$.

En dépit des évènements qui le jalonnent, ce récit ne contient aucune autre référence à la durée ; les cinq substantifs renvoyant sémantiquement au temps (jour, matin, aube, matinée, regards/instants) n'inscrivent aucun fait, aucune situation, dans une temporalité objective :

- Le substantif «jour» (yawm) apparaît au moins une fois dans les différentes versions du texte : « le jour noir » (al yawmu l-'aswadu), expression par laquelle le fils de Bišr répond à la question : «Qui es- tu, fils d'aucune mère ${ }^{26}$ ?» (man 'anta lā'umma laka). Dans certains manuscrits «jour» apparaît également comme nous l'avons vu dans : «Je n'ai jamais vu de jour semblable à celui-ci ». Il convient de souligner que dans l'un et l'autre cas, le mot employé figurément ne désigne pas prioritairement « un espace de temps ».

- Le substantif «matin» (subḥ) est utilisé métaphoriquement par l'épouse quand elle invite

\footnotetext{
${ }^{24}$ Souligné par nous.

${ }^{25} \mathrm{mq}$., p. $25^{2}$.

${ }^{26}$ Nous avons retenu, ici également, le sens littéral d'une expression lexicalisée qui, selon le contexte exprime une malédiction ou un compliment. Il apparaîtra plus loin que c'est le sens propre de lā 'umma laka qui doit être pris en considération dans ce texte (cf. 3-2-2).
} 
Bišr à la comparer à Fāṭima : «(...). Et si Bišr mesurait ses appas à l'aune des miens, le matin se lèverait pour celui qui a des yeux pour voir $\aleph^{27}$, lui dit-elle, indiquant ainsi que l'incontestable supériorité de la beauté de Fāțima ne saurait échapper à un regard avisé.

- Le substantif «aube» (făgr $)$ est employé par Bišr lorsque, dans un vers, il décrit l'épée étincelante qu'il brandit contre le lion : «J'agitai devant lui mon épée et crus vraiment qu'en la dégainant j'avais arraché une aube au cœur des ténèbres) $»^{28}$.

- Le substantif «matinée » (gadāt) est employé dans le poème lorsque le héros, cherchant à impressionner le lion, évoque une ancienne bataille par lui remportée grâce à l'épée : « Ce que firent ses tranchants à Kāẓima, la matinée durant laquelle je rencontrai 'Amr, ne te serait-il donc pas parvenu ? ${ }^{29}$. Il est bien question ici d'un laps de temps mais d'un temps étranger au déroulement de la fiction et qui ne l'inscrit en aucune façon dans la temporalité.

- Il convient de souligner enfin que le substantif lahazāt dont il n'est pas possible de déterminer à quelle date précise il prit dans le lexique arabe le sens de «moment, instant», apparaît ici sans équivoque possible dans son sens premier de « regard ». Décrivant le lion qui lui fait face, Bišr précise : «Fier, il montrait ses griffes, ses crocs acérés et des regards que l'on aurait pu croire des braises $»^{3^{\circ}}$.

Pas plus que ces substantifs, les termes «puis» $(f a)$ et «ensuite» (tumma) ne marquent clairement une chronologie. Ainsi, les huit occurrences de «ensuite» (tumma) marquent chacune un changement scénique, le glissement d'un état à un autre, d'une situation dans une autre, constituant un enchaînement allégorique non nécessairement chronologique.

Avant d'analyser l'importance particulière que revêt le verbe donner un délai ('amhala), seule trace dans le texte d'une véritable durée, il est intéressant de commencer par aborder deux exemples qui illustrent particulièrement bien l'indifférence de cette maqāma au temps réel, à la chronologie évènementielle.

Le premier exemple concerne le problème posé par la lecture de la séquence consonantique 'qret ses implications, dans :

«Il rencontra le lion et son poulain se cabra. Il mit pied à terre et ' $q r / a h u$. Ensuite, dégainant son épée ${ }^{31}$ en

\footnotetext{
${ }^{27} \mathrm{mq}$., p. 25 .

${ }^{28}$ mq., p. 255.

${ }^{29}$ mq. , p. 254.

${ }^{30} \mathrm{mq}$., p. 254.

${ }^{31}$ Souligné par nous.
} 
direction du lion, il lui coupa la route et le pourfendit $»^{3^{2}}$.

Si l'on considère que 'qr renvoie à la deuxième forme verbale, 'aqqara, on lit: 'aqqara muhrahu soit « il maudit son poulain ». Dans ce cas, le texte ne pose aucun problème.

La compétence de Muhammad 'Abduh est incontestable pour ce qui concerne la connaissance du lexique de la langue arabe classique et de sa tradition grammaticale. Dès lors, il serait pour le moins hasardeux d'attribuer la lecture qu'il propose et les commentaires embarrassés qu'elle lui inspire à une quelconque ignorance.

Par contre, la lecture que propose Muhammad 'Abduh conduit à une apparente inversion de l'ordre des événements :

«Il rencontra le lion et son poulain se cabra. Il mit pied à terre et lui trancha les jarrets avec son épée ${ }^{33}$. Ensuite, dégainant son épée en direction du lion, il lui coupa la route et le pourfendit $»^{34}$.

En effet, son embarras devant cette inversion (Bišr fait usage de son épée, puis la tire de son fourreau) est manifeste dans ces quelques remarques :

« Il lui trancha les pattes avec son épée signifie : il lui faucha les pattes avec une épée. Il dégaina son épée en direction du lion signifie : il tira son épée du fourreau et s'avança vers lui. La signification de ces expressions fait apparaître qu'il n'a tiré son épée que pour s'avancer vers le lion. Pourtant, c'est avec elle et rien d'autre qu'il a tranché les pattes du poulain. En réalité il a voulu dire ${ }^{35}$ qu'après avoir tranché les pattes du poulain, ayant donc « pour cela » dégainé son épée, il s'avança vers le lion ; non pas qu'il aurait à nouveau dégainé après avoir tranché les pattes avec une épée. On pourrait peut-être entendre par trancher avec une épée : lier, ligoter car cela ressemble au fait de faucher les pattes ; l'un et l'autre en effet empêchent de marcher. $»^{36}$

Substituant à ce que l'auteur a écrit ce qu'il le suppose avoir voulu écrire, Muhammad 'Abduh propose de contourner cette apparente contradiction par deux moyens : soit en attribuant à «trancher les pattes avec une épée » ('aqara) un sens figuré, hypothèse qu'il relativise ; soit en « reclassant» les événements du texte dans une chronologie cohérente : il suppose pour celà à « dégainer » ('ihtarața) une antériorité qu'il n' a pas dans la maqāma et transforme l'action exprimée par ce verbe en état, lui attribuant le sens de «ayant dégainé » (muhtaritan).

\footnotetext{
${ }^{32}$ mq., p. 252. « fa-mā nașșafa-hu hattā laqiya l-'asada wa-qamașa muhru-hu fa-nazala wa-'aqara-hu țumma hutarața sayfahu 'ilā l-'asadi wa- 'taraḍa-hu wa-qațta-hu».

Notre lecture de cet extrait, comme celle de M. 'Abduh, prend le verbe 'ihtarața dans sa signification de « tirer l'épée de son fourreau ». André Roman attire notre attention sur le fait qu'il serait possible que ce verbe ait signifié, dans l'usage : « retourner»; cette signification s'inscrit en logique continuité avec celle de la racine HRT: « tourner » mais elle n'est pas attestée par Ibn Manzūr. Ce qui oblige à en chercher trace dans des réalisations littéraires, tâche que nous n'avons pu accomplir. Il va de soi que si les textes de l'époque confirmaient l'utilisation de 'ihtarata pour «retourner», l'explication de M. 'Abduh et notre commentaire seraient à revoir.

${ }^{33}$ Souligné par nous.

${ }^{34} \mathrm{mq}$. p. 252.

${ }^{35}$ Souligné par nous.

${ }^{36} \mathrm{mq}$., p. 252, note 8 .
} 
Dans la même perspective, on pourrait considérer cette inversion comme un procédé rhétorique par lequel Hamadānīin indique que le lion seul méritait que Bišr dégainât.

Par-delà les considérations philologiques et, en tout état de cause, la lecture de Muhammad 'Abduh demeure une excellente illustration de l'effet produit sur le lecteur par l'absence de temporalité dans le texte.

D'ailleurs, de même que Muhammad 'Abduh tente de replacer le texte dans une dimension « chrono-logique », les copistes paraissent avoir été troublés par l'a-temporalité qui marque cette maqāma puisqu'ils ont tenté de la modifier. C'est en effet une des variantes proposées dans certaines versions de l'œuvre qui constitue le second exemple que nous allons maintenant examiner.

Cette variante se situe au moment où Bišr est éconduit par son oncle. La version la plus répandue est la suivante :

« [Bišr] fit serment de ne pas cesser de les harceler s'il ne lui donnait sa fille en mariage. Ensuite, ses méfaits à leur encontre se multiplièrent. Les préjudices qu'il leur portait ne discontinuaient pas. » ${ }^{37}$

\section{Certains manuscrits proposent :}

[Bišr] fit serment de ne pas cesser de les harceler s'il ne lui donnait sa fille en mariage. Ensuite les jours se trainèrent, les nuits se déroulèrent, les mois se succédèrent, les années s'écoulèrent, tandis que Bišr attaquait sans relâche ceux d'entre eux qu'il rencontrait ${ }^{38}$, Ses méfaits à leur égard se multiplièrent. Les préjudices qu'il Ses méfaits leur égard se multiplièrent. Les préjudices leur portait ne discontinuèrent pas. $»^{39}$

Il est vrai qu'une telle accumulation de détails, dont l'abus chez certains auteurs vaut à la prose rimée médiévale sa réputation de redondance, n'est pas, en elle-même, étrangère au style de Hamad̄ānī. Cependant, abstraction faite des idées reçues à ce sujet, l'auteur est l'un de ceux qui, le plus souvent, en font usage à bon escient, lorsque cette accumulation informe le texte et éclaire le lecteur. Que l'on pense par exemple à l'hôte dans la célèbre al-maqāma almadīiriyya ${ }^{40}$. Ses propos suffocants et ses discours verbeux, faits de répétitions inutiles accumulées, campent mieux l'insupportable personnage qu'une longue et minutieuse description. Mais, dans al-maqāma al-bišriyya rapide, concise, incisive, cette énumération, qui reste sans écho dans le texte, détonne. Rompant avec l'unité stylistique, brisant une

\footnotetext{
${ }^{37} m q$., p. 251-252.

${ }^{38}$ Souligné par nous.

${ }^{39}$ mq., p. 251-252, note 6 : wa-yurwā ba 'da qawlihi 'in lā yuzawwiğhu bnatahu țumma dabbati l-ayyāmu wa-darağati llayālī wa-tașarramati š-šuhūru wa-tağarramati s-sunūnu wa-bišrun yaftuku fì man laqiyahu minhum wa-katurat madarratuhu ilà 'âhirihi.

${ }^{40} m q$. , pp. 104-118.
} 
atmosphère étale où le temps est suspendu, déversant dans le texte en quelques mots juxtaposés toute la durée qui lui manque, elle paraît avoir pour principale fonction de situer l'apparition ultérieure du fils de Bišr dans la logique du réalisme.

Il est difficile d'attribuer à l'auteur lui-même cette rupture probante dans l'écriture du texte. Ceci reviendrait à lui imputer le choix délibéré, ou maladroit, de désagréger l'unité structurelle de la maqāma en essayant d'en combler d'un jet les lacunes chronologiques, comme s'il avait ignoré, lui qui joue précisément de ce fait, que la logique de l'apparition ultérieure du fils de Bišr s'explique de manière cohérente par la nature de la filiation spirituelle et non génitale qui les unit.

Tandis que les différents éléments que nous venons d'examiner contribuent à confirmer le caractère éminemment accessoire de la temporalité dans ce texte, le verbe «accorder un sursis » ('amhala) introduit singulièrement dans le récit la dimension du temps. Ce délai que l'oncle réclame afin de «faire périr par quelque moyen», est cependant un délai d'un caractère particulier, n'échéant qu'avec la mort hypothétique du héros, un espace ouvert, étranger à la détermination de la durée, limité par son début, indéterminé quant à son terme. Selon notre hypothèse, la mort dont il est ici question n'est pas la mort physiologique mais symbolique, celle qui est curieusement commune à toutes les initiations et qui marque le passage au statut d'initié. La mort de Bišr, venant mettre fin à ce délai, ne serait pas son décès mais son accès au savoir qu'il quête. Cette explication ne peut prendre son plein sens isolément, c'est pourquoi après l'avoir signalée nous en examinerons l'importance et la cohérence dans la suite de cet article (voir notamment $§ 3-2$ ).

1.4. Le chemin, les lieux

L'espace de la maqāma est moins topologique qu'allégorique : les lieux cités dans le texte présentent tous une résonance particulière avec des concepts religieux ou ontologiques et ne circonscrivent aucune topologie reconnaissable (fictive ou réelle).

Les substantifs qui désignent un lieu sont tous utilisés au sens figuré, à l'exception, combien éloquente, de terre ('arḍ) et de chemin (țarīq).

Terre (deux occurrences) renvoie notamment à l'espace de la Quête : le monde que doit parcourir celui qui veut acquérir et transmettre la gnose. Mais dans la pensée ismaïlienne ou 
«Dieu n'a créé aucune chose en ce monde... sans que cette chose propose un symbole ${ }^{41}$, les symboles renvoient sans cesse à des «variantes et additions ${ }^{42}$ et l'on peut également dire, sans qu'une explication entraîne l'exclusion de l'autre, que «la Terre en son immensité symbolise avec l'héritier spirituel du Prophète $»^{43}$.

Le substantif chemin $(\operatorname{tar} \bar{q})$ est dérivé de la racine $T R Q$ comme le terme țarīqa (réalisation obligatoire en cas de pause du terme țaríqat $)^{44}$. Tarīqa «a pris deux acceptions techniques successives en mystique musulmane (...) c'est une méthode de psychologie morale pour guider pratiquement les vocations individuelles $»^{45}$ puis, dans un second temps, ce terme a désigné également les confréries, congrégations et écoles mystiques. Outre la proximité sémantique et phonétique de tarī et țarīqa deux faits en accentuent les liens dans le texte.

- Sur les trois occurrences de tarī q, le substantif est à deux reprises utilisé au « cas direct déterminé » soit at-țarīa et se réalise donc comme l'homophone de tarīqa. Dans la perspective qui nous requiert, cette homophonie renforce le lien complexe qui existe entre țarīq et țarīqa.

- La permutation dans la maqāma du prénom de Fāțima (une occurrence) et de sa forme tronquée «Fāțim» (une occurrence) favorise des permutations similaires, celle que nous venons de relever ( țarīq / țarīqa) et d'autres qui seront signalées ultérieurement.

Les termes : air/espace sol (haḍị̣̂) et déserts (falā) ne sont pas utilisés ici dans leur sens de lieu ou d'étendue :

- Air/espace $(\breve{g} a w w)$ est utilisé dans l'expression lexicalisée halawti ğawwan, littéralement : « sois vide en ton espace », que nous avons rendue par «sois esseulée ».

- Sol est utilisé par Bišr quand il évoque son honneur perdu, « tombé aussi bas que le sol».

- Désert est utilisé dans l'expression lexicalisée «un fils des déserts- ('ibnun li-l-falā) métaphore désignant le serpent et dont l'intérêt tient à la relation qu'elle évoque entre le serpent, dont il est question ici, et l'héritier de Bišr qui est lui aussi, comme nous le verrons, un fils du désert.

Il convient d'indiquer, en ce qui concerne les substantifs ci-dessus énumérés, que Hamad̄ānī

\footnotetext{
${ }^{41}$ Corbin H., op. cit., p. 12.

${ }^{42}$ Ibid.

${ }^{43}$ Ibid.

${ }^{44} L A$, II, țaraqa, pp. 585-589.

${ }^{45}$ Massingon Louis, « Ṭarīqa », EI, IV, pp. 700-705 ; p. 700.
} 
met en œuvre un procédé d'écriture fondé sur un apparent paradoxe : pour mettre en lumière la dimension allégorique de certains termes (ici : terre, chemin), il les emploie précisément dans leur sens propre, dans un contexte où tous les autres termes appartenant au même champ sémantique sont utilisés au sens figuré. Ainsi, «terre » et «chemin » ne se distinguent pas seulement en référence à leur sens hors-texte, mais tout autant par le fait qu'ils sont employés au sens propre, ce qui, dans ce texte, les oppose aux autres substantifs désignant un lieu et employés quant à eux au sens figuré.

Par-delà ce remarquable procédé d'écriture, la portée des termes : terre et chemin ne peut être mesurée sans examen préalable des toponymes contenus dans le texte, examen qui permettra d'établir leur imprécision géographique et leur portée symbolique.

Le premier toponyme cite peut être localisé avec précision. Il s'agit de Huzāà (deux occurrences) nom d'une tribu, désignant par extension le territoire qu'elle occupe. C'est à Huzā'a que l'oncle envoie Bišr quérir pour Fāṭima une dot de mille chamelles. Nous n'avons trouvé aucune indication qui put attester que les chameaux élevés par cette tribu se distinguaient particulièrement de leurs congénères.

À l'époque où écrivait al-Hamad̄ānī, la tribu de Huzā'a, depuis longtemps installée autour de la Mecque, était surtout renommée pour son «attitude méritoire à l'égard du Prophète ${ }^{46}$. Si le territoire de cette tribu peut être localisé sans difficulté, le fait que le texte ne contient aucune indication sur le point de départ de Bišr, rend cependant impossible une identification du chemin périlleux qu'il emprunte pour s'y rendre ${ }^{47}$. Bien plus, le texte ne fait état que d'un seul chemin que Bišr peut emprunter et c'est précisément parce qu'il ne peut choisir entre plusieurs itinéraires qu'il sera contraint d'affronter le lion et le serpent :

«L'objectif de l'oncle était que Bišr empuntât le chemin qui le séparait de Huzā'a en sorte que le lion le dévorerait ; parce que les Arabes évitaient d'emprunter ce chemin-là sur lequel se trouvaient un lion nommé Don et un serpent appelé Courageux (...) Ensuite, Bišr emprunta ce chemin-là » ${ }^{48}$.

Ces propos ne font allusion à aucune légende dont nous ayons pu trouver trace ou qui soit évoquée par les commentateurs.

Le chemin emprunté par Bišr passe en tous cas par Bațnu Habt, où le héros situe sa rencontre

\footnotetext{
${ }^{46}$ Kister M. J., « $\underline{\text { khuzà'a } », ~} E F^{2}, \mathrm{~V}, \mathrm{pp} .79-82, \mathrm{p} .81$.

${ }^{47}$ Considérer que Bišr emprunte inévitablement un chemin qui mène du territoire de sa tribu ('Abd al-Qays) à Huzā'a ne reposerait sur aucun élément textuel.

${ }^{48} m q .$, p. 254.
} 
avec le lion, dans le poème qu'il compose à l'occasion de son combat avec l'animal. Ce toponyme est inconnu des commentateurs. Ainsi, Muḥammad 'Abduh précise :

«Bațu Habtest peut-être un toponyme mais nous ne le trouvons ni dans les ouvrages qui décrivent les pays ni dans ceux qui décrivent les lieux. $»^{49}$

Dans le souci d'identifier l'endroit, les commentaires proposent de traiter séparément les deux substantifs qui composent le toponyme. Pour qui prend baț dans son sens de «creux», l'expression bațu habt signifie «au creux de Habt»; solution insatisfaisante s'il en est puisque Habt est cité dans quatre toponymes : Certains auteurs situent entre La Mecque et Médine Habt al-bazwā' et Habt al-ğamīš ; Habt serait également l'un des campements de la tribu yéménite de Zubayd et enfin le nom d'un point d'eau appartenant à la tribu syrienne de Kalb. Procédant toujours par déduction, les commentateurs proposent de retenir l'un des deux premiers lieux parce qu'ils se trouvent, à l'instar de Huzāáa, à proximité de La Mecque. Leur analyse ne conduit donc, dans le meilleur des cas, qu'à une identification qui demeure imprécise.

Enfin, Kāzima, dernier toponyme cité dans le texte, désigne une «étape sur la route de Bașra à la Yamāma » ${ }^{50}$.

1.5. Les métamorphoses d'une monture

Pas plus que le temps ou les lieux, les autres éléments du texte n’ont vocation événementielle réaliste ou objective. La monture même de Bišr dont le rôle peut paraître secondaire lors d'une première lecture, illustre bien la liberté du document par rapport au réalisme. En effet, chaque fois que la préoccupation majeure de Bišr se modifie, on observe que le substantif qui désigne sa monture se modifie également de sorte que, par le biais d'une racine commune, il est en relation directe avec le désir qui meut le héros.

Ainsi, lorsque Bišr est requis tout entier par la dot de sa cousine $(\mathrm{MaHR})$, il est monté sur un poulain $(M u H R)$. Lorsqu'ayant surmonté les obstacles, il est devenu digne d'elle, il devra se

\footnotetext{
${ }^{49}$ mq., p. 253 .

${ }^{50}$ Cornu Georgette, Atlas du Monde arabo-islamique à l'Epoque classique, IXe-Xe S. (Répertoire des toponymes des cartes VII à XI- présentation provisoire), Brill et CNRS, Leiden, 1985, t. I, 87 p., p. 13.

Yaqūt, Mu 'ğam al-buldān, éd. Dār Ṣādir, Beyrouth, 1957, vol. 4, p. 430 : « 'alā sayfi al-baḥri fi țarīqi al-baḥrayn mina albașra baynahā wa-bayna al-bașra marhalatayn. » Une étape (marhala) correspondait à une journée de voyage en moyenne et à une distance de 30 miles soit $48 \mathrm{~km}$.

'Abd al-Ḥamīd signale, dans son commentaire des Maqāmāt, un autre lieu également nommé Kāẓima et situé près de Médine. Nous n'en avons pas trouvé mention ailleurs.
} 
mesurer à un chevalier « armé de pied en cap (...)», « un adolescent imberbe éclatant comme un quartier de lune $»^{51}$ et monté sur une jument (FaRaS). Il est vrai que le substantif : chevalier (FāRiS) n'apparaît pas dans le texte. Il y est néanmoins implicitement désigné par cet «adolescent imberbe» et «armé de pied en cap ». Nous verrons plus loi, dans le détail, l'importance de la dimension allégorique du chevalier dans la quête initiatique ismaïlienne. Enfin, lorsque Bišr parvient au terme de sa quête, quand il a transmis à son tour ce qui lui a été transmis, ce « dépôt » ('amāna) représenté dans le texte par la femme vertueuse (HaṢāN ) dont il comprend qu'il ne peut se l'approprier, il prend en même temps l'engagement de ne plus monter à cheval $(H i S ̣ a ̄ N)$.

Poulain, jument ou cheval, la monture de Bišr n'est décidément pas un animal ordinaire. Plutôt que d'exprimer cela par des métaphores ou par des explications circonstanciées, l'auteur a préféré une nouvelle fois utiliser l'opposition des termes dans le contexte. Alors qu'ils sont tous trois utilisés dans leur sens propre, les mots : poulain (muhr), jument (faras) et cheval (ḥiṣān) perdent leur banalité de substantifs usuels par leur relation à dot (mahr), chevalier (fāris) et femme vertueuse (ḥașān).

D'autre part, le texte prête à penser que la monture du héros fut également le support sur lequel il rédigea avec le sang du lion le poème qu'il adressa à sa cousine : « (...) Ensuite, avec le sang du lion, il écrivit sur son qamīṣ à sa cousine $»^{5^{2}}$. Qamīṣ signifie : tunique ; mais il a deux autres significations qui méritent ici l'attention : cheval qui se cabre et, par lexicalisation d'une métaphore utilisée par le Prophète lui-même, Califat ${ }^{53}$, ce qui désigne dans l'Islam ši íte la succession de l'Imam.

Le fait que qamīs, ne renvoie pas seulement à un vêtement mais au cheval, symbole des désirs qui meuvent Bišr - et par-delà le cheval à l'imam - est annoncé par la présence dans le texte, quelques mots plus haut, du verbe qamașa. Ce verbe qui signifie «se cabrer » est celui par lequel est dépeinte la réaction du poulain à la vue du lion. L'adjectif qamīṣ désigne (en rapport avec ce verbe) un animal qui se cabre.

Dans la mesure où la correspondance lexicale entre les préoccupations du héros et le

\footnotetext{
${ }^{51}$ mq. , p. 257 .

${ }^{2} m q .$, p. $25^{2}$.

${ }^{53}$ LA, III, qamaṣa, p. 162 : « al-qamīṣu llad̄ì yulbasu ma 'rūfun (...) 'arāda bi-l-qamīṣi l-huilāfata fi hādāa l-hadịt (...) qamaṣa l-farasu 'an yarfa a yadayhi wa-yațrahahumā ma an wa-ya '̆̌̆ina bi-riğlayhi ».
} 
substantif qui désigne sa monture fait de celle-ci un point central de la dynamique de Bišr, le poème qu'il compose et rédige sur son qamiș doit être à son tour reconsidéré. Ces vers ont pu passer et passent encore pour une pièce de bravoure, aspect accentué par le fait que, isolés de la maqāma, ils ont connu une existence autonome. Examinés non plus en eux-mêmes mais tels qu'ils s'inscrivent dans le document, ils sont riches d'informations multiples concernant l'évolution de Bišr dans sa quête. Les éléments les plus probants ont été abordés ou le seront tout au long de cet article. Mais ce poème mériterait une étude exhaustive et détaillée mettant en évidence la dialectique qui l'inscrit dans une tradition poétique des plus anciennes en même temps qu'il constitue une pièce originale et extrêmement dense.

1.6. Le nom propre des personnages

L'importance que revêtent dans cette maqāma les noms propres des différents personnages tient à trois facteurs: leur signification, leurs connotations et l'opposition entre les personnages dotés d'un nom propre et ceux qui demeurent anonymes.

Il convient, pour commencer, d'exclure de la présente analyse le nom de 'Īsā Ibn Hišām, d'après lequel le narrateur rapporte son récit. Il s'agit là d'un personnage qui est présent dans la phrase inaugurale de chacune des maqāma-s de l'auteur. La signification de son nom ne peut être sérieusement examinée que dans une perspective globale incluant l'ensemble des pièces. En effet, seuls les personnages qui appartiennent spécifiquement à al-maqāma albišriyya retiendront notre attention. Quatre sont désignés par un nom propre : Bišr, Fāțima, le lion Don et le serpent Courageux.

1.6.1. 'Amr, la fonction du nom propre

Dans le poème qu'il compose après avoir rencontré le lion, Bišr fait mention d'un personnage nommé 'Amr, il s'agirait d'un inconnu vaincu par le héros au cours d'un combat. L'homophone du prénom masculin 'Am signifie vie, religion ${ }^{54}$.

L'intérêt de la polysémie des noms propres ne tient pas seulement ici à l'intérêt général de la polysémie dans toute analyse littéraire. Il est lié, de façon plus singulière au statut particulier des noms propres dans la langue arabe puisqu'ils sont, pour la plupart, simultanément noms propres et noms communs gardant leur plein sens dans le lexique, ce qui accentue leur aspect

\footnotetext{
${ }^{54}$ LA, II, 'amara, pp. 881-885. p. 881 : « al- 'amru l-ḥayātu wa-summiya r-ră̆ulu 'amran tafā'ulan an yabqā (...) wa-qīla l'amru d-dìnu. »
} 
éponymique. Soulignons enfin, que la polysémie des noms propres renvoie ici à l'utilisation de la fonction symbolique du nom ${ }^{55}$ dans les rites initiatiques ismaïliens. C'est pourquoi il ne paraît pas abusif de voir dans le combat livré par le héros contre 'Amrl'expression de la lutte qui oppose en lui la religion exotérique, à laquelle il lui faut renoncer, et la Religion ésotérique, qu'il lui faut acquérir (cf. 3-2).

1.6.2. Bišr Ibn 'Awāna al-'Abdiyy

Bišr (vingt et une occurrences) n'est désigné par son nom complet : Bišr Ibn `Awāna al- 'Abdiyy que dans la première phrase du texte. Par la suite, il sera uniquement désigné par son prénom, lequel signifie gaieté. Il est dérivé de $B \check{S} R$ comme les termes : bonne nouvelle ( $b a s ̌ r)$, se réjouir d'une bonne nouvelle ( bašira), annoncer une bonne nouvelle ( baššara) et humanité ( bašar $)^{56}$; ces termes ont une connotation positive. Le prénom, Bišr, relie donc le héros à l'humanité dans son ensemble, humanité que réjouit l'annonce d'une bonne nouvelle (celle de la vérité ésotérique comme nous le verrons).

'Awāna, prénom du père de Bišr, dérive de la racine 'WN. Au sens propre ce substantif a des significations aussi diverses que : grand palmier ou petit hérisson ${ }^{57}$. Son intérêt ici tient moins à sa signification propre qu'au choix que fait l'auteur d'un tel prénom. En effet, désigner le père génital de Bišr par un prénom dont le sens propre ne présente pas d'intérêt particulier, alors que tous les autres noms propres sont saturés de significations diverses, choisir cependant un nom qui se rattache par sa racine à des notions importantes comme 'íāna (secours) mais surtout al-mu iin (le Secourable, l'un des attributs d'Allah) est la marque textuelle du peu d'importance dans laquelle les ésotéristes tiennent le père génital par rapport au maître. Celui-ci, considéré comme le véritable père, par lequel l'initié accède à la mort puis à une renaissance symbolique au savoir ${ }^{5^{8}}$, est le seul support de la filiation vraie.

Quant au nom de relation ('ism nisba) 'abdiyy, construit à partir de la racine ' $B D$, il signifie : se rapportant à un esclave, à un serviteur de Dieu. Ce nom représente ici la kunya du héros, en quelque sorte ce qui le qualifie et qualifie son géniteur. Cette kunya désigne les membres

\footnotetext{
${ }^{55}$ Corbin H., op. cit., pp. 19-23.

${ }^{56} L A, \mathrm{I}$, bašara, pp. 216-217.

${ }^{57} L A$, II, 'awana, pp. 934-935.

${ }^{5}$ Corbin H., op. cit., p. 22 : «il ne peut être un homme libre s'il n'est pas ressuscité d'entre les morts, c'est-à-dire s'il n'a pas reçu l'initiation ».
} 
de la tribu des 'Abd al-Qays ${ }^{59}$. Ce terme renvoie à l'opposition entre «esclave » (' $\left.a b d\right)$ et « libre » (hurr) qui, pour les Ismaïliens, emblématise l'une des oppositions possibles entre

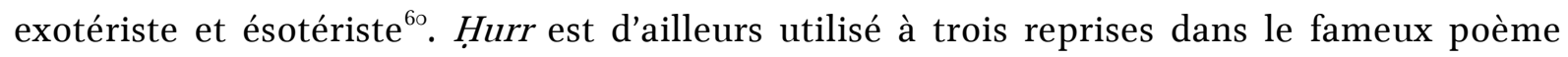
composé après la mort de Don. S'adressant à la dépouille de l'animal, Bišr lui affirme qu'il est «mort libre » (mutta ḥūrran) puis répète à deux reprises qu'il est lui-même un « homme libre»(hurr). L'opposition entre «libre» (ésotériste) et « esclave» (exotériste) ne doit pas occulter l'autre signification du terme 'abdiyy: «se rapportant à un serviteur, un adorateur de Dieu ». Dans la perspective ismaïlienne tout homme, si libre fût-il, demeure soumis à Allah et la liberté que brigue l'impétrant par son initiation demeure l'un des moyens par lesquels il réalisera au mieux son devoir d'adorateur soumis à la force divine.

Dans le nom du héros se concentrent donc plusieurs notions importantes, comme si toute son histoire s'y trouvait à la fois résumée et annoncée.

$$
\text { 1.6.3. Fāṭima }
$$

Par opposition à l'épouse de Bišr qui restera anonyme, sa cousine porte le prénom de Fāṭima (une occurrence). Elle sera également désignée une fois par Fāṭim, forme tronquée de son prestigieux prénom. La valeur symbolique de cette appellation est si éclatante dans l'Islam en général, dans l'Islam ši itite en particulier et singulièrement dans l'Ismaïlisme, qu'il sera examiné dans le cadre de notre étude des grands symboles du ši isme présents dans cette maqāma (cf. 2-1).

\subsubsection{Le lion Don}

Il en sera de même en ce qui concerne la symbolique du lion et sa désignation par Don (trois occurrences). Par contre, nous aborderons ici, le problème d'ordre linguistique que pose ce nom propre. En effet, le terme $d_{a} \underline{d} \underline{q}$ que nous avons traduit par Don n'appartient pas à la langue arabe. Hamad̄ānī, persan arabisé, a choisi là un terme considéré par les Arabes de son temps, comme un terme persan entré dans leur langue par le biais du composé Baǵdād. Selon le lexicographe Ibn Manzūur :

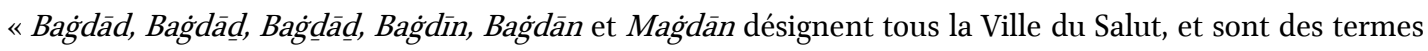
persans. Ils signifient : don d'une idole car Baǵ est une idole et dād et les autres termes équivalents : don. ${ }^{61}$

\footnotetext{
${ }^{59}$ Caskel W., « 'Abd al-Qays », $E F$, I, p. 74-76.

${ }^{6 \circ} L A$, II, 'abada, pp. 664-667.

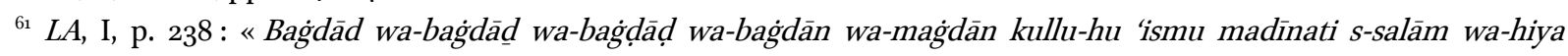
fārisiyya ma 'nā-hu 'ațā'u șanamin li-'anna bağ șanamun wa-dād wa-ahawātu-hā 'ațiyya ».
} 
Si cette explication est aujourd'hui remise en cause, du moins par certains ${ }^{6_{2}}$, il n'en demeure pas moins, et c'est ce qui nous intéresse, qu'elle prévalait du temps de l'auteur, autorisant la traduction de $d \bar{a} \underline{d}$ par Don. En effet, compte tenu de la relation particulière des Arabes à leur langue, il est important que le terme $d \bar{a} \underline{d}$ trouve son sens non pas en référence directe à la seule langue persane mais, tout autant, en référence à la représentation que pouvaient avoir les Arabes de certains mots appartement selon eux à cette langue. Sachant que, par ailleurs, $d \bar{a} \underline{d}$ renvoie aux deux notions de justice et de don par le truchement du terme persan $: d \bar{a} d^{b_{3}}$, la portée du choix de l'auteur apparaître pleinement lorsque nous étudierons sa relation avec le symbolisme véhiculé par la figure de Don ( $c f$. 2-1-2).

\subsubsection{Courageux}

Le dernier nom propre que nous examinerons ici est celui du serpent Courageux (deux occurrences). Le terme choisi par l'auteur comme nom propre du serpent a le double sens de «courageux » et de «serpent ${ }^{64}$. Ce serpent Courageux/Serpent est l'un des indices les plus explicites de la présence dans le texte d'un jeu d'analogies ( $c f . \S 1-7)$, sorte d'emboitement de miroirs déformants qui instaure des relations de similitude entre les différents personnages en même temps qu'il sert parfois à renforcer l'un de leurs traits singuliers.

\subsubsection{L'épouse anonyme}

Pour rendre la lecture de cet article plus commode, nous avons désigné la femme du héros, demeurée anonyme dans le texte de Hamad̄ānī, par «l'épouse » ou «l'épouse /la femme de Bišr ». Il est temps maintenant de préciser qu'elle n'est jamais désignée de la sorte dans la maqāma ou elle apparaît comme : «une belle femme que [Bišr] épousa », une «belle aux blanches dents », « (...) la femme qui t'a désigné ta cousine » ou plus implicitement « elle » ou $\ll \mathrm{tu} »$.

Il y a dans le document une contradiction manifeste puisque Bišr qui se marie au début du récit, adresse plus loin à son fils les propos suivants : «Louange à Allah ! Jamais je ne me suis marié avec une noble épouse $! »^{65}$.

\footnotetext{
${ }^{62}$ Duri, « Baghdād», EF, I, pp. 921-936; p. 9 : «le nom est employé avant Hammourabi donc indubitablement à une époque antérieure à une quelconque influence persane ».

${ }^{63}$ Naficy Saïd, Dictionnaire français-persan, Librairie Imprimerie Beroukhim, Téhéran, 1973 ; t. I ; p. 572 ; p. 109 o.

${ }^{64} L A$, šaga 'a, p. 273-274; p. 273 c.

${ }_{5}^{6}$ mq., p. 258 : « yā subḥāna llāhi mā qārantu 'aqīlatan qațțu».
} 
Considérant que la phrase ne prend sens que par un implicite qui serait : « ... pour avoir un fils aussi courageux », certains lecteurs ont pu considérer qu'il n'y avait la aucune contradiction. Cependant, dans la mesure où aucune contrainte d'ordre linguistique n'imposait à l'auteur de faire dire à son héros «jamais je ne me suis marié avec une noble épouse » pour, en fait, remercier Allah de lui avoir donné un fils courageux, nous considérons que cet énoncé, tel qu'il se présente (et non pas tel que l'on pourrait l'interpréter hors-texte pour lui donner une cohérence réaliste) est pour le moins pertinent. Que donc, pris comme tel, il contredit les événements décrits au début du récit.

D'ailleurs, lorsqu'on aborde ce problème non en termes de mensonge, d'illogisme ou de dérobade (de la part du héros, du narrateur ou de l'auteur), mais en termes de choix lexical, on se donne la liberté d'observer qu'il y a dans le document deux racines distinctes à partir desquelles sont construits les différents mots concernant le mariage ; d'une part $Z W G$, d'autre part $Q R N$ :

- De $Z W G$ sont dérivés épouser (tazawwağa), donner en mariage ( zawwă̆, tazwĭğ).

- de $Q R N$ est dérive qarana, verbe utilisé par Bišr quand il nie avoir été marié.

Plusieurs indices examinés tout au long de ce travail attestent que les dérives de $Z W G$ renvoient au mariage en tant qu'allégorie de l'acquisition de la gnose et de sa transmission (cf. 1-3 et 3-2). Dans cette perspective, qārana renvoie pour sa part au mariage considère au sens propre $^{66}$. Le fait que Bišr nie avoir été marié (au sens propre : qārantu) nous confirme dans la certitude que l'ensemble des autres occurrences concernant le mariage sont à prendre figurément ; que la relation qui le lie à la belle qu'il abandonne, à Fāțima qu'il espère et au jeune adolescent qui se présente comme son fils, n'est pas une relation qu'il convient de comprendre dans son sens ordinaire.

$$
\text { 1.6.7. Les «personnages-archétypes }{ }^{67}
$$

Comme l'épouse, l'oncle du héros reste anonyme. Selon H. Corbin, c'est le propre du maître dans le cheminement initiatique ${ }^{68}$ et, effectivement, lorsque nous esquisserons une lecture

\footnotetext{
${ }^{66}$ L'édition de Constantinople présente, sur ce point, plusieurs variantes dont l'intérêt ne peut être mis en évidence sans une étude portant spécifiquement sur cette version.

${ }^{67}$ Corbin H., op. cit., p. 18-19 : « peu importe que leurs noms à l'un et à l'autre ne nous soient pas dévoilés ; notre roman ne met en scène ni des allégories, ni des figures imaginaires ou mythiques, mais des personnages-archétypes ».

${ }^{68}$ Corbin H., op. cit., p. 2 : « Il y a le sage (al- âlim) (...); son rôle est de personnifier parfaitement le dâ îismaélien, et c'est pourquoi sans doute aucun nom propre ne lui est donné ».
} 
portant sur l'ensemble de la maqāma, il apparaîtra que tel est bien le rôle de ce personnage. Le fils du héros, enfin, ne porte pas de nom propre. De même que l'oncle personnifie celui qui peut transmettre la gnose, le fils de Bišr représente celui à qui elle peut être transmise.

Rappelons que, sommé de se présenter, le fils du héros dit : «je suis le jour noir et la mort rouge ». Qu'il s'agisse là de deux expressions lexicalisées n'empêche pas de reconnaître dans « la mort rouge » l'expression qui désigne « chez les mystiques [la] résistance de l'homme à ses passions $»^{69}$ et dans «le jour noir » la référence à un jour de deuil ou de tristesse. S'il est vrai que la couleur noire fut, historiquement, la couleur des Abbassides, elle semble avoir occupé dans la symbolique une place un peu particulière en tant que «non-couleur », par opposition au rouge, au jaune, au vert et au blanc. Mais les couleurs sont ici, par-delà leur fonction symbolique (cf. 1- 7-3), l'un des supports des analogies que nous allons maintenant évoquer.

1.7. Quelques analogies remarquables

Ces réseaux d'analogies portant sur divers éléments lexicaux, syntaxiques ou rhétoriques, se cristallisent autour du personnage de Bišr, à partir duquel ils s'organisent et se distribuent.

\subsubsection{Bišr est Don}

Nous avons déjà relevé la similitude établie entre le héros et le lion par l'adjectif « libre ». D'autres indices étoffent cette ressemblance : Bišr assure à sa cousine qu'il est «un lion visitant un lion » ((...) laytan zāra laytan), « un lion énorme et invincible rencontrant son semblable » (... hizabran 'áglaba lāqā hizabran) ; il dit au lion mort : « Mon cœur est semblable au tien » (wa-qalbī mițlu qalbika) puis déclare «j'ai tué l'un de mes pairs » (qataltu munāsibî̀). Ces comparaisons sont, certes, loin d'être originales dans la littérature arabe, pas plus que ne le sont d'ailleurs le combat héroïque d'un homme contre un lion ou la volonté affirmée par certains de voir mourir un tiers dans semblable combat. Ainsi, par exemple, l'histoire de Ğahdar Ibn Malik combattant dans une arène, par ordre d'al-Hağğăğ Ibn Yusuf, un lion affamé $^{70} n^{\prime} e s t$ pas sans présenter dans sa trame et l'esprit qui la sous-tend, des similitudes avec le triangle: Bišr-Don-l'oncle. De même, les vers composes par Ğahdar à cette occasion ${ }^{71}$

\footnotetext{
${ }^{69}$ Biberstein Kazimirski A. (de), Dictionnaire Arabe-Français, Maisonneuve et cie, Paris, 1860 ; rep. Librairie du Liban, Beyrouth, s.d., 2 tomes 22 x 14,5, t. 1, 1392 p. ; t. 2, 1638 p. t. 2, p. 1165 .

${ }^{70}$ Al-Suyūṭī, Šarḥ šawāhid al-mug̉nī, lağnat al-turāt al-'arabī, Dār Maktabat al-Ḥayāt, s. l., s. d. ; 2 vol. pp. 407-411.

${ }^{71}$ Ibid.
} 
confirment que Hamad̄ānī s'inscrit bien, en tout état de cause, dans la tradition littéraire de son temps et qu'il s'y inscrit d'autant mieux que le lion continuera d'inspirer d'autres poètes après lui.

1.7.2. Bišr « est » serpent

Semblable au lion, Bišr l'est aussi au serpent. C'est encore dans le poème que nous rencontrons un premier indice. Bišr, affirmant avec fierté qu'il est «un homme d'ascendance irréprochable », utilise 1'expression lexicalisée $\underline{d} \bar{u}$ țarfayni $i^{2}$ qui désigne notamment une espèce de serpent. Plus loin, après avoir tué Courageux, le héros déclame : «son être est mon être, mon venin son venin » (wa-nafsu-hu nafsī wa-sammī sammu-hu).

Comme le héros, son fils est également serpent. Avant de se faire connaître, le jeune homme se présente comme étant « le jour noir » (al-yawmu l-'aswadu). Or, I'adjectif 'aswad, signifiant noir, désigne également, par métonymie, une sorte de serpent.

Plus tard, lorsque le jeune homme indique qu'il est le fils de Bišr et de «la femme qui [lui] a désigné [sa] cousine », le héros se demande «qu'enfanterait un serpent sinon un serpent ?» ( hal talidu l-ḥayyatu 'illä l-ḥayya).

Cette dernière réplique met au rang de serpent le héros, son « épouse » et son fils.

1.7.3. Les couleurs

Le parallèle entre l'épouse et le fils du héros est confirmé par l'utilisation des noms de couleurs. Si la richesse symbolique de ces noms est particulièrement importante, comme le montrent les longues rubriques que leur consacre Ibn Man ẓūr ${ }^{73}$, nous nous contenterons de les examiner ici dans leur dimension strictement textuelle.

Quatre couleurs sont présentes dans le texte, dont deux de façon patente dans les expressions «le jour noir » et « la mort rouge ». Ces deux noms de couleur apparaissent dans la dernière partie et sont employés dans les deux expressions consécutives par lesquelles le fils de Bišr se présente. Ils font écho à deux énoncés que l'on rencontre au début de la maqāma : évoquant l'esseulement futur auquel il condamne son épouse en l'abandonnant, le héros lui dit : «fașfirī wa-bị̣̂ī. Cette expression a pour signification, littéralement : «sens-toi vide et dessèchetoi », mais Hamad̄ānī utilise ici l'impératif des verbes șafara et bāḍa qu'on pourrait traduire

\footnotetext{
${ }^{72} L A$, II, țarafa, pp. 582-585, p. 585 : « al-'aswadu dū țt-tarfayni hayyatun».

${ }^{73}$ LA, II, sawada, pp. 233-236 ; LA, I, hamara, pp. 774-717 ; LA, I, bayada, pp. 295-298 ; LA, II, șafara, pp. 448-450.
} 
également : « jaunis et blanchis $»^{74}$.

Pour chacun de ces deux personnages, les deux éléments constituant chaque paire de noms de couleur sont très proches l'un de l'autre dans le texte.

1.7.4. Bišr et son oncle

Le parallèle entre Bišr et son oncle est marqué par deux traits. Tout d'abord, l'un et l'autre font un serment et semblent ne pas le tenir : l'oncle a «fait le serment» de ne donner sa fille en mariage qu'au prétendant qui lui offrira une dot de mille chamelles ; Bišr a fait, lui, le serment de ne cesser de harceler le clan jusqu'à obtenir la main de sa cousine. Le verbe utilisé dans les deux cas est le même : 'āla. Or, le héros cesse de harceler le clan pour aller quérir les chamelles et l'oncle accepte de lui donner sa fille alors qu'il n'a pas réuni le troupeau demandé.

Un autre verbe, halafa, est utilisé dans la dernière phrase pour signifier faire un serment. L'opposition 'āla/ halafa n'est pas sans rappeler l'opposition qarana/ zawwağa. 'Āla désigne des engagements non tenus (dans leur aspect patent) et halafa un engagement tenu, celui du héros à l'égard du dépôt confié.

Autre similitude entre l'oncle et le neveu : l'un et l'autre empruntent le chemin menant à Huzā'a, chemin symbolique qui est au cœur de la maqāma.

Les éléments qui renforcent les liens tissés entre les différents personnages humains et animaux, accentuant leurs ressemblances par-delà leur individualité, sont encore nombreux. Après avoir signalé les plus remarquables, il est maintenant nécessaire d'établir comment l'ensemble des indices que nous venons de décrire (a-temporalité symbolique des lieux, noms propres, métamorphoses de la monture de Bišr, analogies entre les personnages) contribuent à organiser une épopée mystique.

2. Al-maqāma al-bišriyya, une épopée mystique

2.1. Les grands symboles du šíisme

Comme nous l'avons indiqué plus haut, il est notoire que Hamaḍān̄̄ fréquentait assidûment les milieux ismaïliens. Mais l'Ismaïlisme ne saurait être tenu pour monolithique et uniforme, c'est pourquoi nous ne traiterons dans cette première partie que d'éléments unanimement reconnus comme symboles fondamentaux de ce courant et dont la présence dans

\footnotetext{
${ }^{74}$ Nous ne retenons pas les autres significations possibles de ces deux verbes, dans la mesure où elles ne s'inscrivent pas dans le cadre de notre démonstration que d'ailleurs elles ne confirment ni n'infirment.
} 
la maqāma est confirmée clairement par le texte. Rappelons rapidement que «pour l'essentiel, le chi'isme oppose, à la réalité historique du pouvoir califien assuré par les Umayyades et les Abbassides, un ordre idéal de succession du Prophète, celui de la légitimité dynastique des imâms alides, investis de décret divin ou en vertu d'une désignation explicite, par l'Imâm vivant de son successeur $»^{75}$. Pour les Ismaïliens, cet « imâm se présente comme un personnage souffrant, un témoin douloureux de la foi $»^{76}$.

Afin de limiter notre approche aux grands symboles ismaïliens, nous excluons délibérément de notre analyse ce qu'il est convenu de désigner par les « universaux symboliques». Si ces universaux ont un rôle certain lorsque l'on s'interroge sur ce qui structure l'humanité, il n'est en rien surprenant que Hamaḍānī et la langue qu'il emploie en témoignent. Ce fait ne nous paraît pas cependant devoir conférer à notre étude une quelconque dynamique.

2.1.1. Fāțima, parangon de la beauté humaine

Objet inaccessible du désir de Bišr, mais objet qui légitime son désir, et par là même restaure sa dignité, la figure de Fāṭima occupe évidemment une place essentielle dans ce texte où elle n'apparaît pourtant qu'à travers ce qui est dit d'elle. Seule femme nommée dans la maqāma, elle y est présentée comme «la plus belle d'entre les humains » ('aḥsanu man yamšī 'alā riğlayni). Absente physiquement du récit mais point focal de toute l'intrigue, elle est le seul personnage à être mentionné dans le discours de tous les autres.

Fāṭima fut, rappelons-le, la fille du Prophète Muhammad, l'épouse de son cousin 'Alī Ibn 'Abī Ṭālib, la mère des deux martyrs al-Ḥasan et al-Ḥusayn. Elle est une figure essentielle de l'Islam dont la présence excède considérablement le rôle historique. Surnommée «mère de son père » ('ummu 'abībā), ainsi exclue de la généalogie et du temps, elle sera considérée très tôt dans l'histoire, comme l'incarnation exemplaire de la compassion. Plus encore, elle acquerra une envergure archétypique dont Louis Massignon définit avec précision les principaux aspects. Ainsi, elle est parfois considérée comme la «mère prééternelle de deux Élus destinés à une mort cruelle pour la justice $»^{77}$, comme «otage humain de l'affirmation de

\footnotetext{
${ }^{75}$ Miquel A., L'Islam et sa Civilisation, Destins du monde, VIIe-XXe siècles, ed. A. Colin, Paris, 1968, 572 p. ; p. 100

${ }^{76}$ Miquel A., op. cit., p. 101

${ }^{77}$ Massignon L., Parole donnée, Julliard, Paris, 1962, 10-18, Paris, 1970, 505 p. pp. 148-170 : «La mubāhala de Médine et l'hyperdulie de Fatima» ; p. 168.
} 
l'inaccessibilité divine $»^{78}$. Elle sera même nommée « du nom d'un attribut divin : Fāțir $»^{79}$. Certains «projettent dans la gloire de la prééternité » «son couronnement céleste ${ }^{80}$. Pour d'autres, elle est «la Caverne d'Asile ${ }^{{ }^{81}}$ ou « la mosquée el-Aqsa de Jérusalem $»^{{ }_{2}}$.

Fāțima elle-même, tout ce qui la concernait par ailleurs, à commencer par son prénom, furent pour les gnostiques objet de culte et de mystère, matière à divination, arithmomancie et interprétations. Les quelques exemples cités ci-dessus permettent de souligner à quel point il est impensable que Hamad̄ānī ait pu, en toute «innocence », forger une Fāṭima modèle, vertueuse et inaccessible, qui ne fût pas l'évocation de celle qu'il ne pouvait manquer de connaître et de vénérer.

\subsubsection{Le lion Don}

Peu de figures sont, autant que celle du lion, le support des symboles les plus divers. Le lion est, bien sûr, dans de nombreuses cultures, symbole de force et de courage, traits que l'on retrouve évidemment dans ce texte. Il y est «le maître des fauves » (sayyidu s-sibāî) dont le cœur (qalb) «ne craint pas les assauts furieux » (lā yaḩšă mușāwala). Mais la place du lion dans ce texte excède largement ces quelques lieux communs. C'est «le lion d'Allah » ('asadu llāh $)^{8_{3}}$ surnom donné par les šî́ites à 'Alī, l'époux même de Fāṭima, le gendre du Prophète et surtout le premier imam, le premier «détenteur de lumières secrètes reçues de Muhammad » ${ }^{84}$ ' $\mathrm{Alī}$, que certains calligraphes représentent sous cette forme léonine ${ }^{85}$.

Symbole du premier imam, le lion est également, pour les ésotéristes, le « gardien du trône ${ }^{86}$; non pas ce trône dans lequel les anthropomorphistes voient le siège (polymorphe) sur lequel Dieu serait « assis ${ }^{87}$ mais le trône allégorique désignant le « savoir absolu de Dieu » ou « son royaume ${ }^{88}$. Ainsi, le lion barre l'accès à ce qui, par-delà la gnose, demeure et doit demeurer «l'obscur objet du désir humain ».

\footnotetext{
${ }^{78}$ Massignon L., op. cit., p. 166.

${ }^{79}$ Ibid.

${ }^{80}$ Massignon L., op. cit., p. 167.

${ }^{81}$ Massignon L., op. cit., p. 168.

${ }^{82}$ Ibid.

${ }^{8_{3}}$ Kindermann H., «Al-'Asad », EI , I, pp. 702-704; p. 7 O3 b : « Les shi'ites appellent 'Alī b. Abī Ṭālib le "lion de Dieu" ».

${ }^{84}$ Miquel A., op. cit., p. 100.

${ }^{85}$ Massoudy H., Calligraphie arabe vivante, Flammarion, 1981, 192 p. ; p. 118-119.

${ }^{86}$ Kindermann H., op. cit, p. 703 b. Huart CI., Sadan J., « Kursi », EI , V, p. 512-513.

${ }^{87}$ Huart CI., Sadan, op. cit., p. 512.

${ }^{88}$ Ibid.
} 
Enfin, en choisissant de désigner l'animal par un nom d'origine persane, Hamadāānī fait, non sans humour, référence à sa seconde culture. La Perse, « empire du lion et du soleil $»^{89}$, creuset du šíisme, a le lion pour emblème.

Le lion dans cette maqāma, c'est également un nom propre : Don. Le don, désigne le dépôt confié, transmis de maître à disciple, chaque disciple ne trouvant son accomplissement qu'en devenant à son tour le maître d'un nouvel initié.

\subsubsection{Le serpent}

À l'époque médiévale, le serpent était perçu par les Arabes comme un animal légendaire qui ne mourait jamais de mort naturelle ${ }^{90}$.

La symbolique du serpent ne se limite pas aux aspects que nous allons évoquer. Nous nous arrêterons essentiellement à sa désignation par le terme hayya (sept occurrences). Mais il est certain que sa forme longitudinale n'est pas sans référer à (l'alif initial (...) isolé, sans ligature $»^{91}$, lettre première dans la graphie du nom d'Allah, forme qui aurait constitué, selon H. Corbin, un symbole essentiel chez les ésotéristes ismaïliens. Forme qu'il est difficile de ne pas signaler quand on sait quels enchevêtrements de symboles nourrissaient en grande partie la pensée ésotériste.

Il convient de noter que Hamaḍānī utilisera également (une occurrence) le terme 'afá '́̄ (vipères) et (une occurrence) la figure lexicalisée «fils du désert » ('ibnun li-l-falā) qui désigne le serpent.

Hayya signifie «serpent» et «vivante», il dérive de la racine $H Y W$ dont dérive : «vie » (hayāt). Hayyle masculin du terme hayya a dans la langue des ésotéristes une double fonction essentielle. La première est commune à tous les Musulmans : al-hayy(Le Vivant) est l'un des attributs d'Allah. Mais le mot «vivant»comme le mot «libre » (que nous avons évoqué plus haut) désigne l'homme parvenu à la vérité de la gnose et du savoir ismaïlien. C'est celui qui est passé de la mort de l'ignorance à la re-naissance de l'initiation. Ce masculin a dans le texte une seule occurrence. Il y est utilisé dans une autre de ses significations, celle de «clan» dans l'expression «les hommes du clan» (riğălu l-ḥayy).

\footnotetext{
${ }^{89}$ Kindermann H., op. cit., p. 703.

${ }^{90}$ Ruska J., « Hayya », EF , III, p. 344-45.

${ }^{91}$ Corbin H., op. cit., p. 131.
} 
Il paraît essentiel de souligner que si, à la différence des termes : Fāṭima et Fāṭim, ḥayya et ḥayy (féminin/masculin) ne sont pas permutables, ils sont tous deux présents dans le texte et s'inscrivent dans la liste des termes couplés que Fāṭima et Fāțim autorisent à rapprocher (cf. 1-2).

2.1.4. Les hommes du clan

En réponse aux exactions de Bišr, «les hommes du clan se réunirent avec son oncle et lui dirent : "éloigne de nous ton fou" ». Certains manuscrits proposent une autre version : «(...) et lui dirent ; “mets-nous à l'abri de ses méfaits et accorde-lui ce qu'il désire" » ${ }^{92}$. Dans ces deux versions, il est intéressant de noter qu'aucune mesure n'est directement envisagée par le clan à l'encontre de Bišr : nulle demande de vengeance, nulle sanction collective. Plutôt que de jouer ici le rôle de censeurs qui leur incomberait si le propos de l'auteur tenait uniquement au texte manifeste, les hommes du clan sont ceux qui, percevant l'oncle comme responsable de son neveu, le reconnaissent comme tel et lui accordent une investiture et un pouvoir qui lui permettront de guider le héros qu'ils désignent comme : «ton fou » (... mağnūna-ka).

Comme la signification de hayynous y autorise, il paraît possible de considérer ces « hommes du clan » (riğālu l-hayy) comme représentant également les hommes «vivants » et ceux soumis au Vivant, c'est-à-dire le groupe des initiés qui reconnaissent l'oncle comme l'un des leurs et comme le guide de Bišr.

$$
\text { 2.1.5. Le fou }
$$

Il est intéressant de rappeler rapidement, puisque l'une des versions du texte désigne le héros comme «fou», que le fou, plus précisément «le fou de Layla» (magnūn Laylā), ce poète amoureux, possédé par l'amour à en être dépossédé de lui-même, fut considéré par de nombreux mystiques comme l'exemple de l'homme que sa propre quête de Dieu excède ${ }^{93 .}$

2.2. Une épopée mystique

Si Fāțima est bien dans cette maqāma l'allégorie de la Religion et les péripéties de Bišr, l'histoire de son initiation, puis de celle d'un autre adepte qu'il initie à son tour, ces propos de $\mathrm{H}$, Corbin devront se vérifier dans le récit :

\footnotetext{
${ }^{92}$ mq., p. 252. mq., p. 252, note 2.

${ }^{93}$ Voir notamment: Ibn Monawwar Muhammad, Les Etapes mystiques du Shaykh Abu Sa'id, mystères de la connaissance de l'unique, Unesco, Desclée de Brouwer, Paris, 1970, 410 p.
} 
« Il y a ainsi une double Quête : quête de la gnose qui est celle de la résurrection spirituelle, ensuite quête de celui que le gnostique, à son tour, pourra ressusciter, et qui sera l'héritier légitime auquel il transmettra le Dépôt Confié (...). Conformément à l'idée de la double Quête qui s'impose à l'adepte, le livre comporte en effet deux parties bien distinctes : la première qui est le récit de la Quête de la gnose, s'achève sur une scène d'initiation d'un extrême intérêt, dans l'état actuel de nos connaissances des textes. Commence alors la seconde partie, au cours de laquelle le nouvel initié devient à son tour un maître en gnose (...) et transmet le précieux dépôt à ceux à qui il reconnaît l'aptitude à le recevoir » ${ }^{94}$

2.2.1. La quête du dépôt confié

La première partie de la maqāma commence avec la rencontre de Bišr et de la femme anonyme qu'il «épouse » et prend fin lorsque son oncle lui dit : «Je t’ai exposé [au danger] espérant une chose dont Allah m'a détourné ; reviens donc que je te donne ma fille en mariage ${ }^{95}$. Cette réplique de l'oncle, sur laquelle nous aurons l'occasion de revenir, si elle clôt la première partie par ce qui peut apparaître comme une fin heureuse, est d'autant plus importante qu'elle contient la première occurrence du terme Allah dans le texte.

Il convient de noter que dans cette phrase, le verbe «donner en mariage » (zawwağa) occupe la douzième position dans l'ordre successif des termes. Or, c'est cette même position qu'occupe le verbe «se marier » (tazawwağa) dans la première phrase du récit. Nous avons indiqué précédemment que les dérivés de la racine $Z W \breve{G}$ désignaient moins le mariage pris au sens propre (fonction, dévolue au dérivé de $Q R N$ ) que l'accès à la Religion (cf. 1-5-6).

Nous ne retiendrons de cette cö̈ncidence dans la position des termes que son impact sur l'effet rythmique, refusant de nous engager dans les méandres de l'arithmomancie, sans pour autant omettre de signaler qu'elle occupait chez les ésotéristes une place de choix et qu'il est vraisemblable qu'elle ait quelque fonction dans cette maqāma. Il faudrait, pour en mesurer l'exacte influence, une recherche d'une autre nature que celle qui nous requiert.

Cette première partie retrace, sous couvert de la conquête de Fāțima, le cheminement de Bišr jusqu'au statut d'initié.

- Fāțima, l'épouse virtuelle, exceptionnellement belle, inaccessible pourtant - malgré le caractère légitime et, qui plus est, préférentiel du mariage avec la cousine germaine - est la Religion.

- La dot que l'oncle réclame pour accorder la main de sa fille représente par son caractère

\footnotetext{
${ }^{94}$ Corbin H., op. cit., p. 139.
}

${ }^{95} \mathrm{mq}$., p. 257. 
exorbitant le lourd tribut que le néophyte doit payer pour mériter l'accès à la gnose. Le chiffre mille ('alf), outre sa valeur numérique stricte, « est presque synonyme d'innombrable $»^{96}$. Mille chamelles, c'est beaucoup plus de chamelles qu'on n'en peut compter, davantage qu'il n'est d'usage d'en réclamer pour dot. Le renoncement de l'oncle à toute dot confirme bien qu'il ne s'agissait pas tant de réunir véritablement un immense troupeau que de mettre l'accent sur la valeur incommensurable de ce que Bišr briguait.

- Le rôle de l'oncle dans la première partie de la maqāma peut paraître contradictoire, si on prend au sens propre son projet de faire périr son gueux de neveu. Cependant, cette volonté s'explique différemment si l'on considère l'aspect particulier de cette mort, c'est-à-dire le fait que, pour les Ismaïliens, l'accès à la gnose est une nouvelle naissance qui fait suite à une mort symbolique : «il ne peut être un homme libre s'il n'est pas ressuscité d'entre les morts, c'està-dire s'il n'a pas reçu l'initiation $»^{97}$.

- La belle anonyme que Bišr prend pour épouse représente ici la religion, celle des non initiés. Malgré sa beauté, elle ne peut rivaliser avec Fāțima ; de même que la religion, malgré sa valeur, maintient dans l'ignorance et ne peut concurrencer la Religion. D'ailleurs, le terme ğāhiliyya, qui désigne habituellement les époques antérieures à l'Islam comme ère d'ignorance ( $\breve{g} a h l$ ), est utilisé par les gnostiques pour définir tout temps qui précède l'accès à la gnose. La religion telle qu'on l'entend habituellement s'intègre, selon eux, dans cette ère d'ignorance puisque aussi bien : « la mort spirituelle, c'est l'inconnaissance et l'inconscience, l'agnôsia ( ğahl). La résurrection, c'est s'éveiller de cette inconnaissance par l'éveil à l'ésotérique $»^{98}$ Or, le terme ğăhiliyya est utilisé par l'auteur dans la première partie du texte. En voyant apparaître son oncle, Bišr qui est face au serpent, est saisi du «sentiment anté-islamique de son honneur » (hamiyyatu l-ğāhiliyya). Ce sentiment, plus anté-gnostique qu'anté-islamique, le saisit au moment où il va affronter Courageux. La scène se déroule en présence de l'oncle et renvoie, selon nous, à la dernière phase du cheminement initiatique, celle de l'investiture proprement dite de l'initié, de son accès rituel au cercle de ses semblables. Cette entrée est vécue comme renversement, retournement, bouleversement. Ce que le texte rend par le fait que l'unique occurrence de ğāhiliyya précède immédiatement la confrontation du héros et du

\footnotetext{
${ }^{96}$ Littman E., « 'alf layla wa-layla », E/2, I, p. 369.

${ }^{97}$ Corbin H., op. cit., p. 22.

${ }^{98}$ Corbin H., op. cit., p. 3 .
} 
serpent, tandis que la première occurrence du substantif Allah y fait suite.

Notons d'ailleurs que lorsque l'oncle retrouvera son neveu face au reptile, il attendra que leur combat prenne fin pour dire au héros qu'il lui accorde la main de sa fille : décision dont le texte laisse à penser qu'elle fut pourtant prise auparavant, lors de la réception du poème. Ce qui confère au combat le caractère d'une étape ultime permettant au héros de réaliser son objectif. S'il est vrai qu'en vainquant le lion, il est devenu digne de Fāțima, elle ne lui sera accordée qu'après son intronisation.

Les réseaux d'analogies établis par le texte entre Bišr et Don impliquent une relation d'équivalence telle qu'en tuant son protagoniste, qui est aussi son alter ego, le héros meurt à lui-même. Dans le même temps, par le truchement des mêmes processus d'identification, sa survie est aussi la survie du lion. Leur combat marque le succès du héros dans les épreuves qui lui donnent accès au savoir ésotérique.

Avant de revenir plus synthétiquement sur ces différents points, il convient d'examiner quelques éléments laissés dans l'ombre jusqu'ici.

Comment évaluer, dans la perspective que nous proposons, les intentions, en apparence malveillantes, que l'oncle de Bišr paraît nourrir à l'encontre de son neveu ? Rappelons la phrase qui clôt la première partie de la maqāma: «Je t'ai exposé [au danger] espérant une chose dont Allah m’a détourné ; reviens donc que je te donne ma fille en mariage ».

Cette première traduction correspond à l'acception habituelle de cette phrase, elle s'autorise d'une part de la lecture traditionnelle de l'énoncé lui-même, d'autre part du recours à deux phrases du texte qui semblent la corroborer.

La phrase : «Accordez-moi un délai afin que je le fasse périr par quelque moyen » peut être comprise, comme nous l'avons signalé (cf. 1-3), en référence à la mort symbolique précédant la naissance initiatique. Elle ne peut donc être considérée comme une irréfutable justification des intentions criminelles de l'oncle.

La phrase : wa-nadima 'alā mā mana'ahu tazwiğahā, habituellement comprise comme : « [L'oncle] fut saisi de remords pour l'avoir empêchée de l'épouser » ne résiste pas davantage à l'examen. Aussi nous proposons-nous de montrer comment elle peut être comprise et traduite par: «l'oncle fut saisi d'une grande tristesse en raison de ce qui l'avait [jusqu'ici] empêché de la marier ». 
Soulignons d'une part qu'au verbe nadima ${ }^{99}$, habituellement reçu dans cette phrase comme « être saisi de remords », Ibn Manẓūr donne pour première signification : 'asifa ${ }^{100}$ : «être exagérément triste ou en colère » ('al-mubālaǵatu fì l-ḥuzni wa-l-ǵadab). Notons d'autre part que le fragment de texte : 'alā mā mana 'ahu tazwīğahā n'a pu être compris comme : «pour l'avoir empêchée de l'épouser » qu'en raison de l'interprétation qui est faite de l'élément mā. Les commentateurs ont en effet estimé que cette particule correspondait à ce que les grammairiens désignent par mā l-mașdariyya, c'est-à-dire une particule qui, selon eux, quand elle précède le verbe transforme l'ensemble particule-verbe en mașdar (soit l'équivalent le plus proche, en langue arabe, de l'infinitif de la langue française). Dans cette perspective, 'alā mā mana 'ahu tazwīğahā signifie : « Pour le fait de l'avoir empêchée de l'épouser ». Cependant, mā mana 'ahu signifie également : «ce qui l'a empêché », si l'on voit dans mā le pronom qu'il est de fait ( 'ism mawșū $)$.

On peut donc considérer la lecture que nous proposons comme aussi fondée linguistiquement que celle admise jusqu'ici. Est-il d'ailleurs étonnant que l'initié, au moment où il a trouvé un disciple et s'apprête à lui transmettre le dépôt confié prenne la mesure de la tâche qui lui incombe? Le maître en gnose $\left(d_{\bar{a}} \hat{\imath}\right)$ « n'est pas un missionnaire qui prêche sur la place publique ou à la mosquée $»^{101}$, il doit choisir avec une grande rigueur parmi les «nombreux prétendants », celui à qui il convient de léguer cet héritage spirituel, « sinon il s'exposerait à livrer à celui qui n'en est pas digne le dépôt qui lui a été confié ${ }^{102}$; et si nombreux que soient les prétendants, les disciples restent rares.

Envisagé dans cette perspective, l'énoncé : wa-nadima 'alā mā mana'ahu tazwīğahā ne conditionne plus la lecture de la phrase qui clôt la première partie du texte, et dans laquelle on avait pu trouver l'expression d'un comportement réparateur de l'oncle se sentant coupable d'un grave méfait. L'oncle a certes exposé son neveu au danger mais ce risque est moins son fait que l'inéluctable conséquence du chemin choisi par Bisr.

Il est maintenant possible de s'interroger sur le sens exact de la phrase : Je t'ai exposé [au danger] espérant une chose dont Allah m'a détourné ; reviens donc que je te donne ma fille en

\footnotetext{
${ }^{99}$ LA, III, nadima, p. 6o9-610, p. 609 : « nadima 'alā š-šay'i 'asifa».

${ }^{100}$ LA, I, 'asifa, p. 61-62 ; p. $61 \mathrm{c}:$ : al-'asafu l-mubālaǵatu bi-l-huzni wa-l-gadab»

${ }^{101}$ Corbin H., op. cit., p. 3 .

${ }^{102}$ Ibid.
} 
mariage » ('arraḍtu-ka țama 'an fì amrin țanā llāhu 'inānī 'an-hu).

Examinons pour commencer 'arraḍtu-ka țama'an. Si l'on considère que țama' signifie « avidité » tout autant que «désir ardent», « espoir vif ${ }^{103}$ tandis que le substantif 'amr désigne «une chose», «une affaire quelle qu'elle soit», mais aussi «un ordre, un commandement »-et dans le lexique technique mystique (ou simplement en théologie) «un commandement divin $»^{104}-$, on peut alors comprendre l'expression țama 'an fí amrin comme « ayant le désir ardent d'obéir à un commandement divin ».

Tanā llāhu 'inānī 'an-hu signifie : «Allah tenant mes rênes m’en a éloigné » (littéralement : Allah a tiré mes rênes de cela). Cette phrase est intéressante à plusieurs égards. Nous y avons déjà relevé la première occurrence du terme Allah. Elle tisse également une similitude nouvelle entre Bišr et son oncle, puisque le désir de l'oncle y prend, comme celui de Bišr (cf. § 1-5), l'apparence d'un cheval; un cheval que ne définissent ni dot, ni cavalier, ni femme vertueuse, mais que guide la main même de Dieu.

La polysémie des termes et la structure syntaxique permettent donc de proposer la lecture suivante : « t'exposer au danger fut, pour moi, désir ardent d'obéir à un commandement divin. Allah qui tient mes rênes m'a ensuite arrêté ».

Dès lors, l'oncle n'est pas ce personnage repentant que les commentateurs décrivent d'abord animé d'intentions pour le moins hostiles à l'égard de son neveu. C'est un homme guidé par Allah dans sa tâche et que la réalité du monde attriste.

Adepte de la religion musulmane (époux d'une très belle femme), Bišr découvre que cette religion peut excéder ses propres limites (il apprend de la bouche même de son épouse l'existence de la belle Fāțima). Il s'engage alors dans la quête de la gnose, guidé par un maître sans complaisance, après que la fermeté de son désir a été mise à l'épreuve (après avoir longtemps harcelé le clan, il s'engage, pour obéir à son oncle, dans un chemin périlleux pour quérir mille chamelles en dot). Il subit les épreuves et accède au savoir ésotérique (son poulain se cabre ; il lui tranche les pattes puis combat et tue le lion). Après avoir exprimé sa profession de foi, après que son maître en a pris acte, il est intronisé en sa présence et reconnu initié (après avoir composé un long poème adressé à sa cousine et dont l'oncle a pris connaissance,

\footnotetext{
${ }^{103} L A$, II, țama 'a, pp. 714-716; p. 71 : « aț-țama ú diddū l-ya'si »

${ }^{104} L A$, 1, 'amara, pp. 96-99; p. 97 c.
} 
il combat le serpent et le tue en présence de son oncle qui lui accorde la main de sa fille). Au début de la maqāma, Bišr est un gueux et «pauvre il le fut tant qu'il n'avait pas trouvé la gnose $»^{105}$. L'ayant trouvée, « pauvre il l'est encore tant qu'il n'a pas trouvé le disciple à qui en transmettre le dépôt $»^{106}$. Toutefois, il a acquis tous les caractères du chevalier. Le chevalier, comme le montre H. Corbin, constitue un concept fondamental de la lecture ismaïlienne du monde : «il existe un lien essentiel et profond entre la spiritualité chiite et ismaélienne et l'idée de la fotowwat (persan javânmardî), terme qui connote à la fois l'idée de juvénileté spirituelle et de chevalerie $»^{107}$.

La figure du chevalier, si elle apparaît explicitement sous les traits du fils de Bisr, «armé de pied en cap (...) adolescent imberbe éclatant comme un quartier de lune », n'en désigne pas moins, tout autant, le héros lui-même, à travers les parallèles et analogies qui le relient au jeune homme, archétype du disciple comme nous l'avons vu. C'est à ce juvénile chevalier que Bišr doit, pour accomplir son propre destin, transmettre ce qu'il a acquis. Avant que cette transmission ne soit parachevée, la pauvreté allégorique (évoquée ci-dessus) s'exprime encore à travers la jactance avec laquelle le gueux Bišr se réjouit d'avoir conquis Fāțima.

\subsubsection{La transmission du dépôt confié}

La deuxième partie du récit présente avec la première deux différences fondamentales. Au niveau formel, elle ne contient qu'un seul vers, la première partie en contenant, pour sa part, trente-neuf. Au niveau événementiel, elle substitue aux deux combats opposant un humain et un animal, deux combats opposant un humain à un autre humain ; ce qui lui confère une simplicité de façade que troublent pourtant, à chaque instant, les étranges circonstances dans lesquelles s'affrontent les deux hommes.

Rempli de fierté, la bouche pleine de jactance, Bišr qui s'en retournait - avec son oncle déclara : « ô mon oncle, j'entends le bruit léger d'un gibier» (yā 'ammu 'innī 'asma u hịssa sayd). Son jeune fils surgissait prêt à se mesurer à lui ${ }^{108}$.

\footnotetext{
${ }^{105}$ Corbin H., op. cit., p. 8.

${ }^{106}$ Corbin H., op. cit., p. 3.

${ }^{107}$ Corbin H., op. cit., p. 142.

${ }^{108}$ L'édition de Constantinople présente une variante qui, par-delà son intérêt stylistique souligne le parallèle entre Bišr et son fils, par l'utilisation répétée du verbe țala'a dans ses deux sens de «pavoiser» et de «surgir »: «Bišr s'en retournait, jactance et fierté plein la bouche, et ne cessa de pavoiser (țala a $)$ que lorsque surgit (tala $a)$ armé de pied en
} 
Cette adresse à l'oncle est la dernière trace dans le texte de sa présence physique. La déclaration de Bišr marque sa séparation d'avec son maître : il est désormais engagé dans la « chasse », la recherche de l'adepte qu'il lui faudra initier. Or, «voilà un jeune adolescent à proximité » (fa-'idāà bi-ğulāmin 'alā qayd).

Les deux combats que se livrent les protagonistes reproduisent, dans cette partie, les deux rencontres de Bišr avec le lion et le serpent. Dans le premier combat, le jeune inconnu, tout en retenant son arme, touchera Bišr de vingt coups de lance avant de lui asséner, lors d'un second duel, vingt coups de plat d'épée. Il est fort probable que le nombre vingt - ou deux fois vingt: quarante -renvoie à une signification précise. Mais, là encore, les hypothèses sont multiples et il serait hâtif, sans un travail global portant sur l'aspect numérique du texte, de renvoyer à l'une ou l'autre de ces interprétations.

Ce qui nous intéresse ici c'est le sens que confèrent à chacun de ces combats les paroles qu'échangent les protagonistes.

La relation du jeune homme et du héros est empreinte de la même violence qui liait Bišr et son oncle. Bišr revendiquait la main de Fāṭima et le jeune homme exige que lui soit livré l'oncle. Avant de s'engager dans un combat avec le héros, il lui dit : «tu es en sécurité si tu livres ton oncle» ('anta fí amānin 'id̄ā sallamta 'amma-ka). Plus loin, après que les deux protagonistes par deux fois se sont confrontés, il dira : «ô Bisr, livre ton oncle et pars en sécurité » (yā Bišru sallim 'ammaka wa-dhab fì 'amān). Mais que signifient ici « livrer l'oncle » et « sécurité » ?

Le terme 'amān qui signifie : sécurité, sûreté, il veut dire également foi, pacte. Il est dérivé de 'MN comme le substantif 'amāna qui désigne le dépôt confié. La différence entre les deux termes tient à la présence ou à l'absence de la marque du féminin singulier. Il en était de même, nous l'avons vu pour d'autres termes (Huzā'/Huzā'a, kāẓim/Kāzima, Fātị/Fātima, țarīq/tarìqa, hayy/ḥayya). Le lien entre ces deux termes se trouve donc renforcé.

L'intérêt de cette proximité est accusé par la relation de ces deux termes à : 'îmān qui, dérivé de la même racine, signifie « foi » et, pour les ésotéristes, « explication allégorique du Coran ». Ainsi, les deux phrases que prononce le jeune homme ne peuvent être restreintes à une

cap sur sa jument, un adolescent imberbe éclatant comme un quartier de lune » (fa-rağa a bišrun yamla'u famahu fahran fa-mā labița 'an țala 'a ḥattā țala a 'amradu ka-šiqqi l-qamar 'alā farasihi muḍăğăgan bi-silāḥihi). 
interprétation de type : «tu auras la vie sauve si tu livres ton oncle sans le défendre comme te l'impose le code familial de l'honneur». Le jeune homme invite également Bišr à lui transmettre sans crainte d'erreur ce que son oncle lui a transmis. Il lui demande donc de l'initier à son tour comme le maître l'a initié.

Les combats que se livrent les deux hommes désignent l'initiation puis l'intronisation du jeune adolescent. Bišr a trouvé à son tour le disciple qui assurera la pérennité du savoir qui lui a été transmis.

Ce savoir étant représenté dans le texte par le mariage avec Fāțima, il est logique que le récit prenne fin quand Bišr « donna en mariage sa cousine à son fils » (zawwağa bnata 'ammihi libnihi). La réponse de Bišr à l'injonction « livre ton oncle » est donc le mariage avec Fāṭima, ce qui confirme que « livrer l'oncle » ne peut être pris uniquement au sens propre.

De plus, si ces propos visaient vraiment la personne de l'oncle, on comprendrait mal sa disparition du récit, plus mal encore le fait que Bišr dispose librement de Fāṭima et la donne en mariage à son fils, c'est-à-dire à celui qui entend porter préjudice au père de cette incomparable femme.

Fils spirituel de Bišr, le jeune homme se présente également comme «le fils de la femme qui t'a désigné ta cousine » ( ... bnu l-mar'ati l-latī dallatka 'alā bnati 'ammika). Ce jeune homme est cependant «fils d'aucune mère ». En effet, les premiers mots que Bišr lui adresse sont : «qui es-tu, fils d'aucune mère» (man 'anta lā 'umma laka). L'expression lā 'umma laka constitue simultanément un éloge et une insulte ${ }^{109}$. Par-delà sa dimension méprisante, cette phrase s'ajoute aux éléments qui expriment l'aspect allégorique de la filiation. De même que Bišr est l'époux de cette femme sans pour autant avoir jamais été marié, de même son fils est le fils de cette même femme sans avoir de mère. Pour ce jeune homme comme pour Bišr, cette femme est la religion (dans son acception habituelle) qui les conduit l'un et l'autre à Fāțima, la Religion dans son acception ismaïlienne.

C'est pour la même raison que les combats qu'ils se livrent l'un à l'autre n'ont pas d'issue fatale : le jeune homme retient les coups qu'il assène au héros afin de ne lui faire aucune blessure. La priorité donnée à la quête et à la filiation spirituelle est accentuée par le peu

\footnotetext{
${ }^{109}$ LA, I, 'amama, pp. 101-107, p. 104 : « al-Layțu : idāa qālati l- 'arabu lā 'umma la-ka fa-huwa-madḥun 'inda-hum ; gayruhu : wa-yuqālu là 'umma la-ka wa-huwa-dammun ».
} 
d'estime dans lequel est tenue la filiation génitale. Les personnages se promettent mutuellement la mort par le truchement de : «puisse ta mère pleurer ta mort » (takalat-ka 'ummu-ka) mais la naissance biologique est assimilée à une défécation, chacun souhaitant à l'autre : «puisse celle qui t'a mis au monde comme on expulse un excrément te pleurer» (țakalat-ka man salaḥat-ka).

La seconde partie de la maqāma décrit donc, en continuité avec la première, la deuxième phase de la quête de Bisr. Parvenu à son objectif, il peut «jurer de ne plus monter à cheval » (ḥalafa lā rakiba ḥiṣānan), s'engager à ne plus chercher l'union avec une femme vertueuse (lā tazawwă̆a ḥașānan).

Plus que sur l'expression d'un renoncement, la maqāma se clôt sur celle d'un accomplissement. 